AES/ PE/ 09-17 I njectivity and Gravity Segregation in WAG and SWAG Enhanced Oil Recovery

23-07-2009 A. Faisal 
Title Injectivity and Gravity Segregation in WAG and SWAG Enhanced Oil Recovery

$\begin{array}{lll}\text { Author(s) } & : & \text { A. Faisal } \\ \begin{array}{l}\text { Date } \\ \begin{array}{l}\text { Professor(s) } \\ \text { Supervisor(s) }\end{array}\end{array} & : & \begin{array}{l}\text { 23 July } 2009 \\ \text { Prof. Dr. W.R. Rossen }\end{array} \\ \text { TA Report number } & : & \text { AES/PE/09-17 } \\ \text { Postal Address } & : & \text { Section for Petroleum Engineering } \\ & & \begin{array}{l}\text { Department of Applied Earth Sciences } \\ \text { Delft University of Technology } \\ \text { P.O. Box 5028 }\end{array} \\ & & \begin{array}{l}\text { The Netherlands } \\ \text { (31) } 152781328 \text { (secretary) } \\ \text { Telephone }\end{array} \\ \text { Telefax } & : & \text { (31) } 152781189\end{array}$

Copyright @ 2009 Section for Petroleum Engineering

All rights reserved.

No parts of this publication may be reproduced,

Stored in a retrieval system, or transmitted,

In any form or by any means, electronic,

Mechanical, photocopying, recording, or otherwise,

Without the prior written permission of the

Section for Petroleum Engineering 


\title{
Injectivity and Gravity Segregation in WAG and SWAG Enhanced Oil Recovery
}

\author{
A. Faisal
}

\begin{abstract}
Gas-injection enhanced oil recovery can recover nearly all residual oil where the gas sweeps. Sweep efficiency in these processes is often poor, in large part because of gravity override of gas. Stone and Jenkins presented a model for gravity override in homogeneous reservoirs, showing that the distance gas and water travel before segregation depends directly on injection rate. In cases where injection pressure is limiting, injectivity is key to overcoming gravity override.

Stone assumed continuous injection of gas and water as a model for WAG (Water Alternating Gas), contending that this is valid as long as slugs mix near the well. This model for co-injection can be extended to relate segregation distance for co-injection processes directly to injection pressure. Injectivity depends on saturations very near the well, however. Therefore, where injection pressure is limiting, this model is pessimistic because injectivity in WAG is greater than in co-injection.

We investigate the increase in injectivity possible with WAG compared to co-injection in 1D and $2 \mathrm{D}$, and the implications for gravity override in 2D, using a range of models for gas and water relative permeabilities. We confirm that the greater injectivity of WAG improves vertical sweep compared to Stone's model when injection pressure is limiting. The greatest improvements occur when slugs violate Stone's assumption: that is, when they are too large to mix fully near the well. The increase in injectivity over co-injection is greater for foam than for WAG without foam, because foam has much lower mobility when gas and water flow together.
\end{abstract}

\section{Introduction}

Oil recovery can be classified as primary, secondary, and tertiary recovery. Primary recovery uses natural energy that exists in reservoir as a drive mechanism to produce oil. These drive mechanisms consist of solution gas, gas cap, water drive, and gravity drainage. After these mechanisms can no longer resist depletion of production then usually water or gas is injected to maintain the reservoir pressure and increase oil production. This stage is called secondary recovery. ${ }^{1}$

Tertiary recovery or enhanced oil recovery (EOR) is an approach that uses chemical, miscible and immiscible gas, or thermal methods not only to maintain reservoir pressure but also to provide a changed interaction between rock and fluids to further reduce residual oil saturation and produce the remaining oil.

The overall efficiency of an EOR process comprises microscopic efficiency and macroscopic efficiency, written as:

$$
E=E_{D} \times E_{V}
$$

where $E$ is overall displacement efficiency (fraction), $E_{D}$ is microscopic displacement efficiency (fraction), and $E_{V}$ is macroscopic volumetric efficiency (fraction).

Microscopic efficiency quantifies recovery on the pore scale. Good miscibility between fluids, decreasing interfacial tension between the fluids, oil volume expansion, and reducing oil viscosity can improve this efficiency. Gas injection is good in giving a high value of microscopic 
efficiency. Immiscible gas injection can give residual oil saturation up to $50 \%{ }^{2}$ while miscible gas injection theoretically can reduce oil saturation to $0 \%{ }^{3}$

Macroscopic volumetric efficiency quantifies the fraction of the reservoir volume contacted by injected fluid. It can be divided to areal and vertical sweep. Areal sweep is influence by injection/production well pattern, reservoir permeability heterogeneity, mobility ratio, and relative importance of gravity and viscous force. Vertical sweep is controlled primarily by gravity segregation caused by density difference, mobility ratio, vertical to horizontal permeability variation, and capillary forces. ${ }^{1}$

Two EOR methods that optimize both efficiencies are WAG (Water Alternating Gas) and SAG (Surfactant Alternating Gas).

WAG is a process of injecting water followed by gas, followed by more water, followed by more gas, etc. The gas mixes with the water ahead of it, which causes a reduction in gas mobility. This mixture is effective in displacing oil to the production well, since the macroscopic sweep efficiency is larger than for gas injection only while microscopic efficiency is still high. This is why WAG can improve recovery factor.

WAG is one method of EOR commonly used nowadays because it can optimize the value of both efficiencies and thus give high recovery. Christensen et. al. ${ }^{2}$ and Hallam et. al. ${ }^{4}$ reviewed WAG application to oilfields worldwide and found increases in recovery ranging from 5 to $20 \%$. Dalen et. al. ${ }^{5}$ reported effective displacement and increasing oil recovery with WAG in the attic zone, a zone at the top of reservoir where oil is accumulated, while Sanchez ${ }^{6}$ provided a selection of technical considerations for managing and monitoring WAG projects.

But the problem is that after some time and distance this mixture is separated by gravity. The gas is separated from the mixture and goes to the top of reservoir and overrides the oil, while water goes to the bottom of reservoir and underrides the oil. This phenomenon usually called gravity segregation.

Gravity segregation itself is a competition between gravity (and density difference) and lateral pressure gradient. It occurs when the injected fluid density is higher or lower than the reservoir fluid density. Gravity segregation leads to early breakthrough of the injected fluid and reduced vertical sweep efficiency.

Stone $^{7}$ and Jenkins ${ }^{8}$ made a schematic of gravity segregation in a reservoir. Fig. 1 shows gravity segregation when gas is injected simultaneously with water into reservoir. Three zones with uniform saturation are formed: an override zone, a thin zone at the top of the reservoir where only gas flows; an underride zone, where water only flows; and mixed zone, where gas and water flow together before segregation occurred. A jump of saturation marks the boundary of these three zones.

They then introduced one powerful analytical equation which determines the point of complete segregation of gas and water, based on a steady-state analytical model for co-injection of water and gas in homogeneous horizontal reservoir.

For a radial reservoir the equation can be written as follows:

$$
R_{g}=\sqrt{\frac{Q}{\pi \times k_{z} \times\left(\rho_{w}-\rho_{g}\right) \times g \times \lambda_{r t}{ }^{m}}}
$$

where $R_{g}$ is point of segregation, $Q$ is total injection rate, $k_{z}$ is vertical permeability, $\rho_{w}$ and $\rho_{g}$ are water and gas density; respectively, $g$ is gravity, and $\lambda_{r t}{ }^{m}$ is total relative mobility at the mixed zone. This equation is derived for co-injection, simultaneous water and gas injection from the same interval in a single well (SWAG), and would mimic alternate injection as long as the cycle is kept short enough to make the water and gas mixed well near the well.

Equation 1 clearly shows us that the radius of segregation can be increased by increasing injection rate or by reducing fluid mobility in mixed zone. 
The assumption that WAG process can be represented as a continuous injection is illustrated in Fig. 2, where we can see a zone near the wellbore where water and gas, injected alternately, mix; beyond this region the zones are the same as in a co-injection process.

Rossen and van Duijn ${ }^{9}$ showed that Eq. 2 is rigorously correct if only these following assumptions are fulfilled:

1. Homogeneous, although possibly anisotropic $\left(k_{v} \neq k_{h}\right)$ reservoir.

2. The reservoir is cylindrical with an open outer boundary. The injection well is completed over the entire vertical interval. The reservoir is confined by sealed boundaries above and below.

3. The system is at steady state, with steady injection of fluids at volumetric rate $Q$ and injected fractional flow of water $f_{w}=f_{w}{ }^{J}$. This implies that any remaining oil in the region of interest is at its residual saturation and immobile.

4. Incompressible phases. No mass transfer between phases.

5. Absence of dispersive process, including fingering, and negligible capillary pressure gradients.

6. Newtonian mobilities of all phases.

7. Immediate attainment of local steady-state mobilities, which depend only on local saturations.

Rossen and Shen ${ }^{10}$ derived an equation to relate injection pressure to radius of segregation in radial reservoir. For cylindrical reservoir, the relationship is written as:

$$
p\left(R_{w}\right)-p\left(R_{g}\right)=\frac{k_{z}\left(\rho_{w}-\rho_{g}\right) g}{2 H k_{h}} R_{g}^{2}\left[\ln \left(\frac{R_{g}}{R_{w}}\right)-\frac{1}{2}\left(1-\left(\frac{R_{w}}{R_{g}}\right)^{2}\right)\right]
$$

where $p$ is pressure, and $R_{w}$ wellbore radius. The second term in brackets may disappear depending on how the height of the mixed zone decreases with increasing distance from well.

Based on Eq. 3, Jamshidnezad ${ }^{11}$ plotted the fraction of injection pressure dissipated and (from equations in Rossen and van Duijn) the fraction of injected gas and water lost from the mixed zone against radial position as in Fig. 3. The assumption that is used in this figure is $R_{g}=500 \mathrm{~m}$ and $R_{w}=0.1 \mathrm{~m}$ while mobility in the mixed zone is uniform. About $85 \%$ of the pressure dissipation happens within $50 \mathrm{~m}$ of the injection well; at this position about $1 \%$ of the injected fluid has left the mixed zone. In other words, pressure dissipation happens mostly nears the injection well, while segregation occurs far from the well.

An injection strategy that might increase mobility near the well and increase injection rate, while still keeping low mobility further from the well, might therefore increase the radius of segregation beyond that for co-injection (Eq. 2). ${ }^{11,12,13,14,15,16}$

Foam is an alternative way to reduce mobility in the mixed zone. Foam can be introduced to the reservoir by steady foam injection (co-injecting gas and water with surfactant) or by injecting gas and water to the reservoir in alternating slugs, like a WAG process but with surfactant in the water. The gas and water with surfactant mix to form foam in the reservoir; this is called Surfactant Alternating Gas (SAG).

WAG or SAG injection is a means of increasing injectivity over simultaneous injection of gas and water. Injectivity itself is defined by flow rate injected at the injection well divided by injection pressure. So, in other words, Stone $^{7}$ and Jenkins's ${ }^{8}$ model may be pessimistic on the advantage of vertical sweep efficiency increase in WAG, that has higher injectivity and near well mobility, over co-injection when injection pressure is limited. This paper examines these advantages.

First, we consider and quantify the increase in injectivity of WAG to co-injection in 1D radial flow model as a function of slug sizes of gas and water, starting with slug size small enough to satisfy Stone's assumption that this fluid would mix near the well, before gravity segregation 
begins (Fig. 2). Next, we quantify the increase of radius of segregation and volume of reservoir that is swept by WAG over co-injection in 2D radial flow where injection pressure is limited. Like Stone and Jenkins we focus on the swept zone at steady state (after all mobile oil has been displaced) as an indicator of sweep efficiency expected during the period of oil recovery. Also like Stone and Jenkins, we consider a homogeneous reservoir as a simple basis for analysis.

\section{Fluid and Reservoir Model}

In this study we model the co-injection and WAG process with the STARS ${ }^{\mathrm{TM}}$ simulator, developed by the CMG (Computer Modeling Group), Calgary, Alberta, Canada.

We use a cylindrical model to represent the importance of mobility near the well to injection rate, pressure gradient at the gas front, and ultimately gravity override. We employ three type of contrasting relative-permeability functions: oil-wet relative-permeability functions taken from the Miocene Kareem Formation, United Arab Emirates ${ }^{17}$ (Fig. 4), with residual oil saturation of 0.29; water-wet relative-permeability functions taken from I-Sand Formation, Argentina ${ }^{17}$ (Fig. 5), with residual oil saturation of 0.225 ; and foam effective relative permeability taken from Renkema and $\operatorname{Rossen}^{18}$ (Fig. 6), with residual oil saturation of 0.15 . In all cases initial gas saturation is zero while oil is present at its residual saturation; thus oil is immobile. There is no mutual solubility between oil, water, and gas.

Foam is able to severely reduce gas mobility; this reduction can be represented as a reduction in effective relative permeability and an increase in gas viscosity. Here for simplicity we lump all effects into the gas relative permeability. The simulator we used in this study assumes that foam will be immediately formed when gas meets water with surfactant. Fig. 7 compares the total relative mobility in the presence of foam as a function of water fractional flow to total relative mobility for gas-water flow in the water-wet and oil-wet cases. Here we can see that foam reduces total relative mobility at intermediate range of water fractional flow more than gas-water flow in the other two cases.

The reservoir has a pie shape and has no-flow boundaries on all sides. Injection is simulated by a vertical injection well at the edge of the reservoir and production by a vertical production well on the opposite edge of the model. Both production and injection wells are perforated over the whole interval.

$\mathrm{STARS}^{\mathrm{TM}}$ represents an injector well within a grid block. We wish to have the entire first grid block represent the injection well. So we give the first grid block a diameter of $0.1 \mathrm{~m}$ and the injection well a radius of $4 \mathrm{~cm}$. To avoid a pressure drop within the first grid block, we give this grid block a horizontal permeability of 10,000 D and zero vertical permeability; thus in effect the first grid block represents an injection well of radius $5 \mathrm{~cm}$. In the rest of the reservoir horizontal and vertical permeabilities are $1,000 \mathrm{mD}$ and $100 \mathrm{mD}$, respectively. In all cases the reservoir porosity is $20 \%$, and capillary pressure is neglected in the simulations.

As stated, the first grid block represents an injection well of radius $5 \mathrm{~cm}$. Since injection pressure varies as logarithm of wellbore radius (Eq. 3), our 10-cm-diameter well in a $300 \mathrm{~m}$ cylindrical reservoir plays the same role as a 20 -cm-diameter well in a $600-\mathrm{m}$ cylindrical reservoir. Preventing segregation out to double the distance would require four times the injection rate, however (Eq. 2) and four times the pressure rise at the well (Eq. 3), with four times the effect of compressibility on fluid volumes. In order to minimize the effects of pressure rise and compressibility, we work with the smaller wellbore and shorter radial distances.

The production well, of radius $4 \mathrm{~cm}$, is located in the outermost column of grid blocks. There was virtually no difference in pressure between that in the outermost grid blocks and that in the production well.

To get a better solution for injectivity we refine the first grid blocks near the injector to a smaller size than in the rest of the reservoir. For the oil-wet case we use 20 grid blocks of radius 
$0.5 \mathrm{~m}$ near the injection well, followed by 340 grid blocks of radius $1 \mathrm{~m}$ each. Thus the outer radius is $350 \mathrm{~m}$. For the water-wet case we use $200.5-\mathrm{m}$ grid blocks near the injection well followed by $2901-\mathrm{m}$ grid blocks. For the foam case we use 2501 -m grid blocks. We use different outer radius in these cases with three types of relative-permeability curves because the radius of segregation that we observe is not the same. For instance, the oil-wet case has a greater radius of segregation so the radius of reservoir is larger than for water-wet case, so for the water-wet case we reduce the reservoir radius to reduce the time required for simulation. In all cases we simulate a $15^{\circ}$ sector of the full cylinder, and a reservoir height of $20 \mathrm{~m}$. For 1D radial simulation there is only one grid block in the $\mathrm{z}$ direction, of height $20 \mathrm{~m}$. For the $2 \mathrm{D}$ simulations, there are 20 grid blocks in the $\mathrm{z}$ direction, of height $1 \mathrm{~m}$ each. Fig. 8 shows the reservoir and well configuration.

The initial reservoir pressure is 165 bars, which is also the pressure maintained at the production well. Injection pressure set at 175 bars. The pressure difference between injection and production-well is small enough that fluids are approximately incompressible.

The fluid properties (except for relative permeabilities) are the same for all cases. Water viscosity is $1 \mathrm{cP}$ and gas (supercritical $\mathrm{CO}_{2}$ ) viscosity (set by STARS) is about $0.0155 \mathrm{cP}$ at reservoir conditions. Water density is $1000 \mathrm{~kg} / \mathrm{m}^{3}$ and gas density is approximately $660 \mathrm{~kg} / \mathrm{m}^{3}$ in the range of pressures present in our reservoirs. Liquid compressibility is set to be zero while gas density, viscosity, compressibility are determined by simulator.

Reservoir and fluid properties are listed in Table 1.

The foam model is similar to Renkema and Rossen. ${ }^{18}$ In this model, gas relative permeability with foam $\left(k_{r g}^{f}\right)$ is reduced from that without foam $\left(k_{r g}{ }^{n f}\right)$ by a mobility-reduction factor as follows: ${ }^{19}$

$$
k_{r g}^{f}=k_{r g}{ }^{n f} \times M R F .
$$

This $M R F$ depends on local surfactant concentration, water saturation and oil saturation, as follows:

$$
M R F=\frac{1}{(1+F M M O B \times F D R Y \times F 1 \times F 2)}
$$

where $F M M O B$ is the reference foam mobility reduction factor. FDRY water-saturationdependent function, $F 1$ depends on surfactant concentration, and $F 2$ depends on oil saturation. In this study we use $F M M O B=5500$.

Foam is assumed to collapse below limiting water saturation, SFDRY. This is modeled by the water-saturation-function FDRY:

$$
F D R Y=0.5+\frac{\arctan \left(S F B E T\left(S_{w}-S F D R Y\right)\right)}{\pi}
$$

where $S F B E T$ regulates the slope of the gas relative permeability curve near the critical water saturation. We use SFDRY of 0.316 and SFBET of 1000 .

Foam also collapses if surfactant concentration in the grid block is less than a certain value, as described in a surfactant-concentration relation $F 1$ as follows:

$$
\begin{array}{rlrl}
F 1 & =\left(\frac{C_{s}}{F M S U R F}\right)^{E P S U R F} & \text { for } C_{s}<F M S U R F \\
& =1 & & \text { for } C_{s} \geq F M S U R F
\end{array}
$$


where $C_{s}$ is the surfactant concentration in a grid block, FMSURF the critical surfactant concentration, EPSURF a parameter that controls the dependence of gas mobility on surfactant concentration. Here we use FMSURF of 0.000791 (half the injected concentration) and EPSURF of 1 .

The effect of oil on the foam model is represented by the oil-saturation function $F 2$ :

$$
F 2=\left(\frac{F M O I L-S_{o}}{F M O I L-F L O I L}\right)^{E P O I L}
$$

where FMOIL represents the critical oil saturation above which foam cannot exist, EPOIL regulates to what extent the foam strength is affected by oil saturations less than FMOIL, and FLOIL is lower oil saturation used. In this study we use FMOIL of 0.2, FLOIL of 0.1, and EPOIL of 2 . Since our oil saturation is 0.15 then the presence of oil will lower foam strength to the factor of 0.25 .

Table 2 list foam parameters that are used here.

In this study we employ fixed injection pressure for both co-injection and WAG. Finding Water Gas Ratio (WGR) in a WAG process then requires a trial-and-error process; in our study, we sought WGR $\sim 1: 2$. So for example in the $1 \mathrm{D}$ water-wet WAG case with $0.008 \mathrm{PV}$ per cycle at fixed injection pressure gas is injected for 3 days and water is injected for 10 days. The WGR for this cycle size is 1:1.8. As cycle size varies among our cases, the value of WGR varies in a narrow interval around the desired value of 1:2. Table 3 shows the variation of WGR in WAG injection at different cycle size for all relative permeability curves in the 1D simulation. Variations were similar in the 2D simulations. Here, we only allow WGR to vary in the range between $1: 1.8$ and $1: 2.2$.

\section{Determining $\mathbf{R}_{\mathbf{g}}$}

In numerical simulation, the output result is distorted by numerical dispersion. Stone ${ }^{20}$ reported that numerical dispersion gives a flattening instead of a declining trend of the gassaturation profile in water and gas flood. Van der Heijden ${ }^{21}$ reported the effect of numerical dispersion on the front of surfactant concentration which resulted in the deviations between simulation and the (exact) fractional-flow solution.

Stolwijk's ${ }^{22}$ simulation results for co-injection showed an effect of numerical dispersion on the radius of segregation $\left(R_{g}\right)$. In simulations of a co-injection process, the position of the fluid front between the mixed and underride zones is smeared, showing a gradual change in parameters (e.g. saturation, fractional flow) causing difficulties in determining $R_{g}$ and volume swept. In particular, the value of $R_{g}$ is overestimated if one takes the furthest advance of mobile gas $\left(S_{g} \geq S_{g r}\right)$ as the boundary of the mixed zone. Stolwijk recommended using a gas saturation corresponding to a gas fractional flow a bit larger than zero as the criterion for the boundary of the mixed zone; she reported that this still gives an overestimate of the value of $R_{g}$.

In this study we determine $R_{g}$ based on three criteria involving water fractional flow:

1. $f_{w}$ slightly above the injected value $\left(f_{w} 10 \%\right)$ :

$$
f_{w}=0.9 f_{w}^{J}+0.1
$$

2. $f_{w}$ halfway between injection point and underride zone $\left(f_{w} 50 \%\right)$, written as: 


$$
f_{w}=\frac{f_{w}{ }^{J}+1}{2}
$$

3. $f_{w}$ slightly below its value in the underride zone $\left(f_{w} 90 \%\right)$ :

$$
f_{w}=0.1 f_{w}{ }^{J}+0.9
$$

We compare the simulation results to Eq. 2, using the three criteria above, and find that $f_{w}$ halfway between injection point and underride zone (Eq, 10) gives more accurate estimates for all three sets of relative-permeability functions.

Also, in some 2D cases we find that in the second row of grid blocks the apparent boundary between the mixed zone and the underride zone extends well beyond the trend of the boundary of the layer below and from the analytical solution of Stone and Jenkins. In these cases we extrapolate the boundary of the mixed zone from the trend set over grid blocks below the second row. For example in Fig. 23 we estimate $R_{g}=136 \mathrm{~m}$ rather than $144 \mathrm{~m}$.

Setting $f_{w}$ to $90 \%$ of the injected value gives too high a value of $R_{g}$, while $f_{w}=10 \%$ of the injected value gives too low a value compare to the analytical model. In the other hand, $f_{w}$ halfway between zero and the injected value gives a value of $R_{g}$ closer to the analytical model. For example in the 2D oil-wet simulation of the co-injection process, $f_{w} 90 \%$ gives $R_{g}$ of $135 \mathrm{~m}$, and $f_{w} 10 \%$ gives $R_{g}$ of $111 \mathrm{~m}$, while $f_{w} 50 \%$ gives $R_{g}$ of $123 \mathrm{~m}$, the closest value to $R_{g}$ of $120 \mathrm{~m}$ from analytical model result. In the $2 \mathrm{D}$ simulation of the water-wet co-injection process, $f_{w} 90 \%$ gives $R_{g}$ of $140 \mathrm{~m}$, and $f_{w} 10 \%$ gives $R_{g}$ of $113 \mathrm{~m}$, while $f_{w} 50 \%$ gives $R_{g}$ of $125 \mathrm{~m}$, the closest value to $R_{g}$ of $121 \mathrm{~m}$ from analytical model result. In steady foam injection, $f_{w} 90 \%$ gives $R_{g}$ of $134 \mathrm{~m}, f_{w} 10 \%$ gives $R_{g}$ of $129 \mathrm{~m}$, while $f_{w} 50 \%$ gives $R_{g}$ of $131 \mathrm{~m}$ slightly lower than analytical solution of $132 \mathrm{~m}$.

From these results we conclude that $f_{w} 50 \%$ (Eq. 10) gives the best estimate of $R_{g}$ for all relative-permeability curves we used in this study, since its result always shows a closer value to analytical result.

We used the same criterion of $f_{w}$ to determine the distance gas travels in the WAG processes. In WAG processes, however, the gas front advances and recedes with the cyclic injection of gas and water. We therefore report the furthest and shortest advance of gas using the criterion in Eq. 10. As a second measure of gas sweep, we report the maximum and minimum amounts of gas in the reservoir during the cyclic injection of gas and water in the WAG processes.

\section{WAG Injectivity in 1D}

We do $1 \mathrm{D}$ simulation prior to $2 \mathrm{D}$ simulation to verify that we do reach a steady-state condition as Stone assumes in his analytical equation (Eq. 2) and to determine how long a period of injection is required to reach the steady-state condition. We then analyze injectivity and the radius of segregation in the 2D model after this steady-state condition has been reached. To find the time when the steady state is reached, we plot gas saturation as a function of time and grid block number.

Fig. 9 represents 1D simulation of the oil-wet case with 1 day of gas injection and 2 days of water injection per cycle. In this case the whole 1D cylindrical region of interest represents the mixed zone. So the end of the reservoir where the production well is located represents the edge of the mixed zone.

Steady state is reached after 81 days (0.108 PV) injection, or after 27 cycles; the cyclic variation of saturation after this point is constant. Fig. 10 is an extended version of Fig. 9 after 303 days (0.404 PV), after the steady state condition is achieved. In this figure (with this color 
bar) we can't see any changes of saturation at the edge of reservoir. The performance in this figure is also quite similar to mixed zone in Figure 2, where the area near the wellbore is an area of gas and of area mixture while the rest of reservoir has similar properties which mark the mixing zone. Fig. 11 represents total relative mobility $\lambda_{r t}\left(\right.$ in $\left.(\mathrm{Pa} . \mathrm{s})^{-1}\right)$ after steady state with the same injection cycle. Fig. 12 shows the magnitude of cyclic variations in $\lambda_{r t}$ in WAG divided by $\lambda_{r t}$ from co-injection. We can't make a trend line here since the result is somewhat scattered by the effect of WGR variation among cases (Table 3). In this figure we can see that Stone's assumption (uniform and unchanging mobility in the mixed zone) is not satisfied exactly for any cycle size. With small cycle size the variation of mobility is small while with big cycle size the variation is bigger. For example, with cycles of $5 \%$ of a pore volume the mobility variation is about $10 \%$ at the outer limit of the region.

Fig. 13 illustrates the cyclic variation in injectivity during WAG injection with 10 days of gas injection and 29 days of water injection, with a cycle size of 0.059 PV. Average injectivity with WAG is bigger than co-injection by a factor of 1.24, although this case violates the assumption of Stone in that mobility varies by $25 \%$ at the outer radius during the WAG cycles (Fig. 12). Fig. 14 shows the increase in injectivity as the cycle size increases. Zero cycle size represents coinjection of water and gas. Increasing cycle size gives more benefit to WAG shown by increasing injectivity although it violates the Stone assumption of uniform and unchanging mobility (Fig. 12). About a $12 \%$ increase in injectivity is possible with small cycles that substantially satisfy Stone's assumption.

Fig. 15 and 16 correspond to Figs. 12 and 14, but for the water-wet relative-permeability curves (Fig. 5). Similar to the oil-wet case, there is an increase in injectivity as the cycle size increases, although larger cycles violate Stone's assumption of uniform and unchanging mobility. About a $13 \%$ increase in injectivity is possible with small slugs that satisfy the condition of uniform mobility far from the well and an increasing to about a 30\% increase with larger slug sizes.

Fig. 17 and 18 correspond to Figs. 12 and 14 for foam injection (Fig. 6). The cycle sizes are not as big as in the two previous cases, but the benefit from further increasing cycle sizes appears to be decreasing already in this range. At small cycle size the increase of injectivity is $50 \%$ over steady foam injection, and $150 \%$ over steady foam injection for bigger cycle sizes that still satisfy Stone's assumption of uniform and unchanging mobility far from the injection well. Fig. 19 compares the variation of injectivity with time for SAG injection with the average injectivity for SAG and steady foam injection. The water injectivity in SAG is greater than in steady foam injection because of the severe reduction in mobility at intermediate water fraction with foam (Fig. 7). There is a brief period of low injectivity at the start of injection of gas or water as that phase displaces the other phase from the near-wellbore region.

\section{Summary of Cases in 1D}

In the two cases without foam, modest increases in injectivity are possible, from about $12 \%$ with slugs small enough to satisfy Stone's criterion to about 30\% with slugs large enough to cause significant variations of mobility at the outer edge of the region of interest. For the foam case the benefit of SAG injection over steady foam injection is greater, about $50 \%$ for small slugs and almost $150 \%$ for larger slugs that still satisfy Stone's criterion. One expects (Eq. 2) that the increase in average injectivity would help in fighting gravity segregation. We test this supposition with 2D simulations as described in the next section. 


\section{WAG Injectivity and Gravity Segregation in 2D}

With fixed injection pressure, WAG injection differs from co-injection of gas and water in three ways:

1. Injected water fraction varies with time

2. Injection rate varies with time (cf. Figs. 13 and 19)

3. Average injection rate is larger (Figs. 14, 15, and 18).

Stone's equation predicts an increase in radius of segregation with increase of injection rate. Varying water fractional flow near the well not only gives a larger average injection rate, but also gives a larger pressure gradient further from the well, where most gravity segregation occurs. Fig. 20 shows pressure distribution at the end of water injection in the water-wet case for a cycle size of $0.106 \mathrm{PV}$. The pressure is largely dissipated near the wellbore. Fig. 21 shows pressure distribution at the end of gas injection in the same case; here pressure is dissipated more gradually as a function of distance from the well. With sufficiently small slugs, saturations and mobility are nearly uniform and constant far from the well, but that region does experience a variation of horizontal pressure gradient brought on by the variation of injection rate (Figs. 13 and 19).

$R_{g}$ in WAG and SAG process is not in a static position at steady state. We find its position advances during water injection and recedes during gas injection. We therefore report both a maximum and minimum value of $R_{g}$. Since the volume of gas in the reservoir also increases and decreases during gas and water injection, this volume is also reported as maximum and minimum. Cycle size, in fraction of $\mathrm{PV}$, is based on the pore volume of the cylindrical reservoir in each case. Since this varies from case to case, cycle size should be considered only for comparison within individual cases.

We address the water-wet case first. Using a cutoff of the $50 \%$ gas fractional flow we get $R_{g}=$ $125 \mathrm{~m}$ for co-injection of water and gas (Fig. 22). At small cycle size, $0.001 \mathrm{PV}$, the minimum $R_{g}$ is $136 \mathrm{~m}$ and maximum is $138 \mathrm{~m}$ (Figs. 23 and 24). The $10 \%$ increase in $R_{g}$ represents an expected increase in volume swept (proportional to $R_{g}{ }^{2}$ ) by about a $20 \%$.

Figs. 25 and 26 show the maximum and minimum values of $R_{g}, 225 \mathrm{~m}$ and $200 \mathrm{~m}$, respectively, for a larger cycle size, $0.034 \mathrm{PV}$. The larger value of $R_{g}$ is perhaps a little misleading, because it represents gas about to enter the override zone rather than a large mixed zone. Nonetheless, it does represent additional formation swept by gas. Fig. 27 shows the maximum and minimum values of $R_{g}$ as a function of WAG cycle size for the water wet case. $R_{g}$ increases by $12 \%$ to $14 \%$ for the smallest cycle shown $(0.001 \mathrm{PV})$ and by a factor of over 2.4 for the largest cycle $(0.106 \mathrm{PV})$. No maximum value is shown for the largest cycle because segregation occurs beyond the production well at $300 \mathrm{~m}$.

Fig. 28 shows the minimum and maximum amount of gas present in the reservoir, as a function of cycle size. The maximum gas volume in place increases greatly as cycle size increases, while the minimum also increase modestly. The smallest WAG cycle size tested gives an increase in gas in place of $19 \%$ to $22 \%$, and the largest cycle size an increase by a factor of 1.88 to 4.31 over co-injection, in agreement with the trend in $R_{g}$.

Fig. 29 shows the variation of injection rate for each phase as cycle size increases for WAG injection.

Figs. 30, 31, and 32 corresponding to Figs. 27, 28, and 29, respectively for the oil-wet case. The smallest cycle size shown in Fig. 30 ( 0.002 PV) gives an increase of $8 \%$ to $10 \%$ in $R_{g}$, and the largest cycle size $(0.196 \mathrm{PV})$ gives an increase by a factor of 2.67 to 2.75 . In terms of volume of gas in the reservoir, the smallest cycle size gives an increase $44 \%$ to $48 \%$ and the largest cycle an increase by a factor of 2.2 to 6.1 .

It remains to distinguish the relative roles of the three differences between co-injection and WAG listed at the start of this section - increasing average injection rate, varying injection rate, and varying injected water fraction - in the increased sweep efficiency in WAG. We take as a base case WAG injection with cycle size of $0.008 \mathrm{PV}$ in the water-wet reservoir, for which the 
maximum and minimum values of $R_{g} 143$ and $140 \mathrm{~m}$; for co-injection in the water-wet reservoir at fixed injection pressure, $R_{g}=121 \mathrm{~m}$. Fig. 33 shows sweep with co-injection at fixed WGR but with a fixed, larger injection rate equal to the average injection rate that is possible with WAG. $R_{g}$ is $140 \mathrm{~m}$, greater than the value for co-injection at fixed pressure but less than the maximum value for WAG at fixed pressure. This larger injection rate would be impossible at fixed WGR and fixed injection pressure of $17.5 \mathrm{MPa}$; the case merely illustrates the advantage of WAG in making a greater average injection rate possible at fixed injection pressure.

To isolate the effect of varying injection rate, we inject at fixed WGR using the injection-rate history of the WAG: that is, injection rates that vary by about a factor of three, as they do in WAG at fixed injection pressure, but with fixed WGR throughout. Figs. 34 and 35 show the minimum and maximum values of $R_{g}, 141 \mathrm{~m}$ and $148 \mathrm{~m}$. Injection pressure during injection at the higher rate (18.8 MPa) substantially exceeds $17.5 \mathrm{MPa}$; this case illustrates the benefit of WAG in allowing periods of high injection rate at fixed injection pressure. This result outperforms all the other cases. This suggests that the benefits of WAG in overcoming gravity segregation arise from raising average injection rate (difference 3 listed above) and allowing periods of even higher injection rate (difference 2); varying injected water fraction (difference 1) makes this possible at fixed injection pressure, but by itself variable injected water fraction is harmful to sweep efficiency. The best case, hypothetically, would involve the benefits of increased injectivity but maintain constant WGR.

The benefits of alternate-slug injection are greater for foam than for water-gas flow. Fig. 36 shows the mixed zone during steady foam injection; $R_{g}=132 \mathrm{~m}$. Fig. 37 shows water saturation with SAG with $0.002 \mathrm{PV} /$ cycle, at the end of gas injection; the profile is very similar at the end of water injection. $R_{g}$ is $178 \mathrm{~m}$, an increase of $38 \%$ in $R_{g}$, implying a $90 \%$ increase in volume swept. $R_{g}$ is larger with larger cycle size. Figs. 38 and 39 show results for $0.034 \mathrm{PV} /$ cycle, with $R_{g}=233$ m. Fig. 40 shows $R_{g}$ as a function of cycle size for SAG. The smallest SAG slug size shown gives an $R_{g} 38 \%$ larger than co-injection, and the biggest slug size gives $R_{g} 81 \%$ larger than steady foam injection. Fig. 41 shows the maximum and minimum volumes of gas in the reservoir as a function of cycle size. For the smallest cycle size shown (0.002 PV) the volume of gas in the reservoir is $88 \%$ larger than for steady foam injection; for the largest cycle shown $(0.034 \mathrm{PV})$ gas in place increases by a factor of 3.19 to 3.34 . Fig. 42 shows how injection rates vary with cycle size in the SAG process.

Eq. 3 purports to relate segregation distance to injection pressure for co-injection processes regardless of the mobility of injected fluids (Rossen et al., 2009). In our cases, $R_{g}$ for co-injection is about $125 \mathrm{~m}$ and $123 \mathrm{~m}$ for the water-wet and oil-wet cases without foam (Figs. 27 and 30) and $132 \mathrm{~m}$ for foam (Figs. 36 and 40). This is remarkably good agreement with theory, given that foam mobility about two orders of magnitude lower in the mixed zone in SAG than mobility in the WAG processes (Fig. 7).

\section{Conclusions}

1. The model for gravity segregation in gas EOR proposed by Stone (1982) and Jenkins (1984) applies to processes with fixed injection rate as long as slugs mix in the near-well region and mobility is uniform further from the well, where most gravity segregation occurs. As extended by Rossen and Shen (2007) to cases with fixed injection pressure, the model is pessimistic for WAG processes because it underestimates injectivity and therefore injection rate.

2. Studies of WAG and SAG foam processes in $1 \mathrm{D}$ radial flow show that it is possible to satisfy the criteria of nearly uniform and constant mobility far from the well with sufficiently small slug sizes. With slugs that satisfy this criterion, injectivity is 10 to $30 \%$ greater than coinjection for WAG and 50-150\% greater for foam in the cases examined. The largest increases in injectivity are for slug sizes that violate the assumption of uniform and constant mobility far 
from the well. The increase in injectivity is a result of creating a region near the well of singlephase phase flow during WAG.

3. Studies in 2D radial flow confirm the benefits predicted from the 1D study. In the two WAG examples examined, increases in volume swept of 19 to $22 \%$ and 44 to $48 \%$ are possible with small slugs, and increases by a factor of 1.9 to 4.3 or 2.2 to 6.1 with larger slugs. For the foam case, even small slugs give an increase by a factor of about 3.3 in volume swept.

\section{Nomenclature}

$f_{w}=$ water fractional flow

$g=$ gravitational acceleration

$H=$ height of reservoir

$k_{z}, k_{h}=$ vertical and horizontal absolute permeability, respectively

$S_{w}, S_{o}=$ water and oil saturation, respectively

$R_{g}=$ distance to point of segregation in cylindrical reservoir

$R_{w}=$ radius of wellbore in cylindrical reservoir

$W G R=$ Water Gas Ratio

$p\left(R_{g}\right)=$ pressure at radial position $R_{g}$

$p\left(R_{w}\right)=$ pressure at injection well

$Q=$ total volumetric injection rate of both phases

$r=$ radial position in cylindrical reservoir

$C_{s}=$ surfactant concentration [wt.\%]

$F M M O B=$ reference mobility reduction factor

FMSURF = critical component mole fraction

EPSURF = exponent for composition

$S F D R Y=$ maximum dryout value

SFBET = reference dryout slope

$F M O I L=$ critical oil saturation

$F L O I L=$ lower oil saturation

EPOIL = exponent for oil saturation

FDRY = water-saturation-dependent function

$F 1=$ surfactant-concentration-dependent function

$F 2=$ oil-concentration-dependent function

$\lambda_{r t}{ }^{m}=$ total relative mobility in mixed zone

$\rho_{w}, \rho_{g}=$ water and gas density, respectively

\section{Acknowledgements}

We thank to Computer Modeling Group for use of the simulator STARS and Core Laboratories, Inc. for permission to use the oil-wet and water-wet relative permeability curves from their instructional materials.

\section{References}

1. Green, D.W., Willhite G.P.: "Enhanced Oil Recovery", SPE Reprint Series, Richardson, Texas, 1998. $2-3,74$.

2. Christensen, J.R., Stenby, E.H., Saukage, A.: "Review of WAG Field Experience", paper SPE 39883, SPE International Petroleum Conference and Exhibition of Mexico held in Villahermose, Mexico, 3 - 5 March 1998. 
3. Lake, L.W.: "Enhanced Oil Recovery”. Englewood Cliffs (NJ), USA: Prentice-Hall, 1989.

4. Hallam, R.J., Ma, T.D., Reinbold, E.W.: "Performance Evaluation and Optimization of the Kuparuk Hydrocarbon Miscible Water-Alternating-Gas Flood", New Developments in Improved Oil Recovery, The Geological Society Publishing House, UK, 1995, 153 - 164.

5. Dalen, V., Instefjord, R., Kristensen, R., "A WAG Injection Pilot in the Lower Brent Formation at the Gulfaks Field", New Developments in Improved Oil Recovery, The Geological Society Publishing House, UK, 1995, 143 - 152.

6. Sanchez, N.L.: "Management of Water Alternating Gas (WAG) Injection Projects", paper SPE 53714, SPE Latin America and Caribbean Petroleum Engineering Conference, Caracas, Venezuela, 21 - 23 April 1999.

7. Stone, H.L.: "Vertical Conformance in an Alternating Water-Miscible Gas Flood", paper SPE 11130, SPE Annual Fall Technical Conference and Exhibition in New Orleans, Louisiana, 26 - 29 September 1982.

8. Jenkins, M.K.: “An Analytical Model for Water/Gas Miscible Displacement”, paper SPE 12632, SPE Fourth Symposium on Enhanced Oil Recovery in Tulsa, Oklahoma, 15 - 18 April 1984.

9. Rossen, W.R. and van Duijn, C.J.: "Gravity Segregation in Steady-State Horizontal Flow in Homogeneous Reservoirs", Journal of Petroleum Science and Engineering 43, 2004, 99 111.

10. Rossen, W.R. and Shen, C.: "Gravity Segregation in Gas_injection IOR," paper SPE 107262, SPE Europec/EAGE Annual Conference and Exhibition in London, United Kingdom, 11 - 14 June 2007.

11. Jamshidnezhad, M., Shen, C., Kool, P., Rossen, W.R.: "Improving Injectivity to Fight Gravity Segregation in Gas Enhanced Oil Recovery", paper SPE 112375, SPE International Symposium and Exhibition on Formation Damage Control in Lafayatte, Louisiana, 13 - 15 February 2008.

12. Shi, J. -X. and Rossen, W.R.: "Simulation and Dimensional Analysis of Foam Processes in Porous Media", paper SPE 35166, SPE/DOE Improve Oil Recovery Symposium in Tulsa, Oklahoma, 21 - 24 April 1996.

13. Shi, J. -X. and Rossen, W.R.: "Improved Surfactant-Alternating-Gas Foam Process to Control Gravity Override", paper SPE 39653, SPE/DOE Improve Oil Recovery Symposium in Tulsa, Oklahoma, 19 - 22 April 1998.

14. Shan, D., Rossen, W.R.: "Optimal Injection Strategies for Foam IOR”, SPE 75180, presented at the $13^{\text {th }} \mathrm{SPE} / \mathrm{DOE}$ Symposium in Improved Oil Recovery, Tulsa, Oklahoma, $13-17$ April 2002.

15. Shi, J. -X. and Rossen, W.R.: "Injectivity and Gravity Override in Surfactant-AlternatingGas Foam Processes", paper SPE 30753, presented at the 1995 SPE Annual Technical Conference and Exhibition, Dallas, Texas, 22 - 25 October 1995.

16. Kloet, M. B., Renkema, W. J., and Rossen, W. R.: "Optimal Design Criteria for SAG Foam Processes in Heterogeneous Reservoirs", SPE 121581, presented at the SPE EUROPEC/EAGE Annual Conference and Exhibition, Amsterdam, The Netherlands, 8 - 11 June 2009.

17. Core Laboratories Inc.: "A course in: Special Core Analysis", Dallas, Texas, 1982, 11-6.

18. Renkema, W. J. and Rossen, W.R.: "Success of SAG Foam Processes in Heterogeneous reservoirs", paper SPE 110408, presented at the 2007 SPE Annual Technical Conference and Exhibition, Anaheim, California, 11 - 14 November 2007.

19. STARS ${ }^{\mathrm{TM}}$ Manual, Computer Modeling Group, Calgary, Alberta, Canada.

20. Stone, H.L.: "A Simultaneous Water and Gas Flood Design with Extraordinary Vertical Gas Sweep", SPE 91724, presented at the 2004 SPE International Petroleum Conference, Puebla, Mexico, 8 - 9 November 2004. 
21. Van der Heijden, T.L.M.: "Modeling of Miscible $\mathrm{CO}_{2}$ Foam Displacements with Oil", MSc Thesis Report, Delft University of Technology, 2009.

22. Stolwijk, G.H. and Rossen W.R.: The Effect of reservoir heterogeneity on gravity segregation in gas IOR”, MSc Thesis Report, Delft University of Technology, 2007.

Table 1. Reservoir and fluid properties used in this study

\begin{tabular}{|ll|}
\hline Oil-Wet Case Reservoir properties & \\
Reservoir size $(r, \theta, z)$ & $350 \mathrm{~m}, 15^{\circ}, 20 \mathrm{~m}$ \\
1D Number of grid blocks & $361 \times 1 \times 1$ \\
2D Number of grid blocks & $361 \times 1 \times 20$ \\
$S_{w i}$ & 0.71 \\
$S_{g i}$ & 0 \\
$S_{o i}$ & 0.29 \\
Water-Wet Case Reservoir properties \\
Reservoir size $(r, \theta, z)$ & $300 \mathrm{~m}, 15^{\circ}, 20 \mathrm{~m}$ \\
1D Number of grid blocks & $311 \times 1 \times 1$ \\
2D Number of grid blocks & $311 \times 1 \times 20$ \\
$S_{w i}$ & 0.775 \\
$S_{g i}$ & 0 \\
$S_{o i}$ & 0.225 \\
Foam-Case Reservoir properties & \\
Reservoir size $(r, \theta, z)$ & $250 \mathrm{~m}, 15^{\circ}, 20 \mathrm{~m}$ \\
1D Number of grid blocks & $251 \times 1 \times 1$ \\
2D Number of grid blocks & $251 \times 1 \times 20$ \\
$S_{w i}$ & 0.85 \\
$S_{g i}$ & 0 \\
$S_{o i}$ & 0.15 \\
Horizontal permeability & $1,000 \mathrm{mD}$ \\
Vertical permeability & $100 \mathrm{mD}$ \\
Porosity & $20 \%$ \\
Fluid properties & \\
Water viscosity & $175 \mathrm{cP}$ \\
Gas viscosity & $0.0155 \mathrm{cP}$ \\
Water density & $1,000 \mathrm{~kg} / \mathrm{m}^{3}$ \\
Gas density & $660 \mathrm{~kg} / \mathrm{m}^{3}$ \\
& \\
Initial condition & $165 \mathrm{bars}$ \\
Reservoir pressure & $50{ }^{\circ} \mathrm{C}$ \\
Reservoir temperature & \\
Injection pressure & \\
Wellbore radius & \\
\hline
\end{tabular}


Table 2. Foam parameters

\begin{tabular}{|ll|}
\hline FMMOB & 5,500 \\
FMSURF & 0.000791 \\
EPSURF & 1 \\
FMOIL & 0.2 \\
FLOIL & 0.1 \\
EPOIL & 2 \\
SFDRY & 0.316 \\
SFBET & 1,000 \\
\hline
\end{tabular}

Table 3. The variation of WGR in WAG injection

\begin{tabular}{|c|c|c|c|c|c|}
\hline \multicolumn{2}{|c|}{ Oil Wet } & \multicolumn{2}{c|}{ Water Wet } & \multicolumn{2}{c|}{ Foam } \\
\hline Cycle size & WGR & Cycle size & WGR & Cycle size & WGR \\
\hline 0.002 & $1: 2$ & 0.001 & $1: 1.9$ & 0.002 & $1: 2.2$ \\
0.004 & $1: 1.9$ & 0.008 & $1: 1.8$ & 0.003 & $1: 2$ \\
0.014 & $1: 1.9$ & 0.022 & $1: 1.9$ & 0.007 & $1: 2$ \\
0.059 & $1: 2.1$ & 0.034 & $1: 2$ & 0.015 & $1: 2.1$ \\
0.196 & $1: 2.1$ & 0.106 & $1: 2.1$ & 0.034 & $1: 2.2$ \\
\hline
\end{tabular}

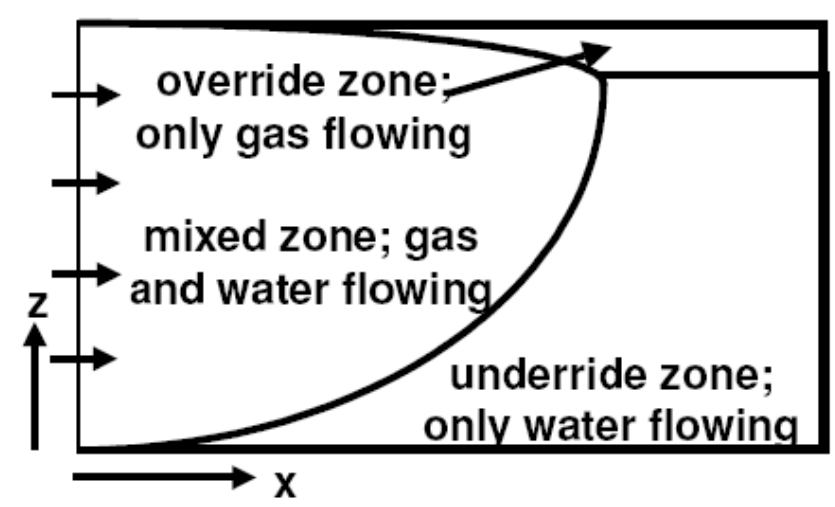

Fig. 1. Schematic of three uniform zones in model of Stone (1982) and Jenkins (1984).

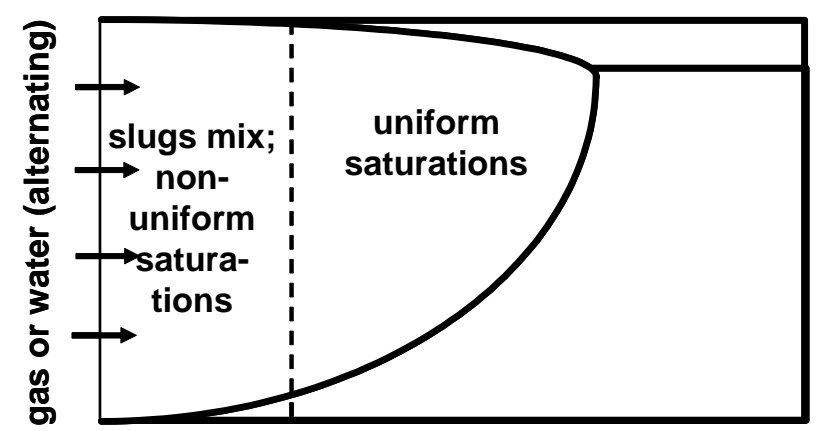

Fig. 2. Schematic of assumption of Stone (1982) and Jenkins (1982) that mixing of slugs in a WAG process occurs in a region near the well that is small compared to the region in which gravity segregation occurs. 


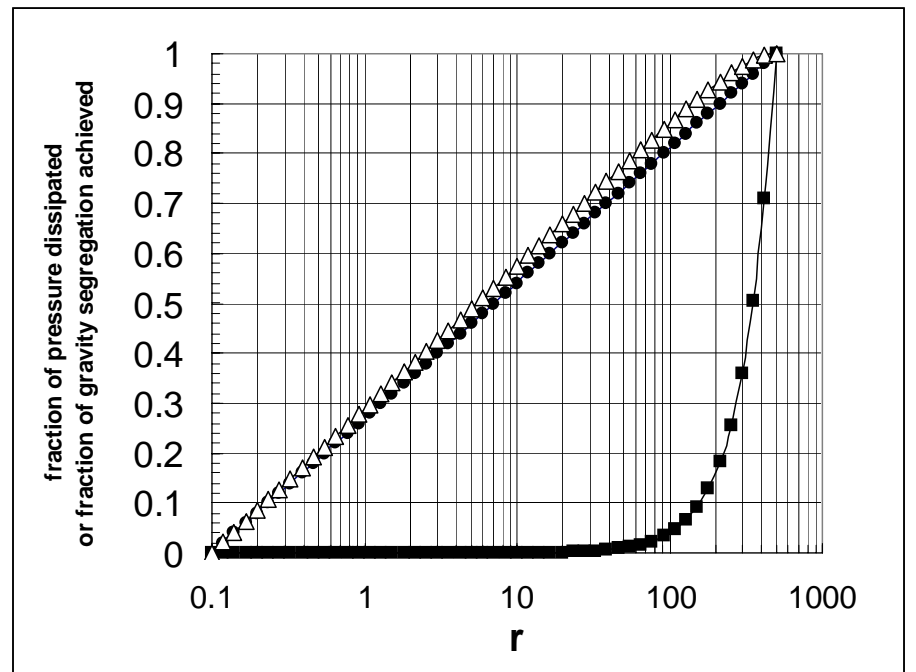

Fig. 3. Fraction of injection pressure dissipated and fraction of segregation of fluids segregated as functions of distance from injection well, in a case where $R_{g}=500 \mathrm{~m}$. The two curves for fraction of injection pressure dissipated reflect different assumptions about the shape of the mixed zone (Rossen and Shen, 2004; Jamshidnezhad et al., 2009). Open triangles: pressure dissipation where the mixed zone doesn't shrink until segregation occurred; closed circle: pressure dissipation where the mixed zone shrinks proportionately to the volume of gas and liquid; closed square: loss of fluids. From Jamshidnezhad et al. (2009).
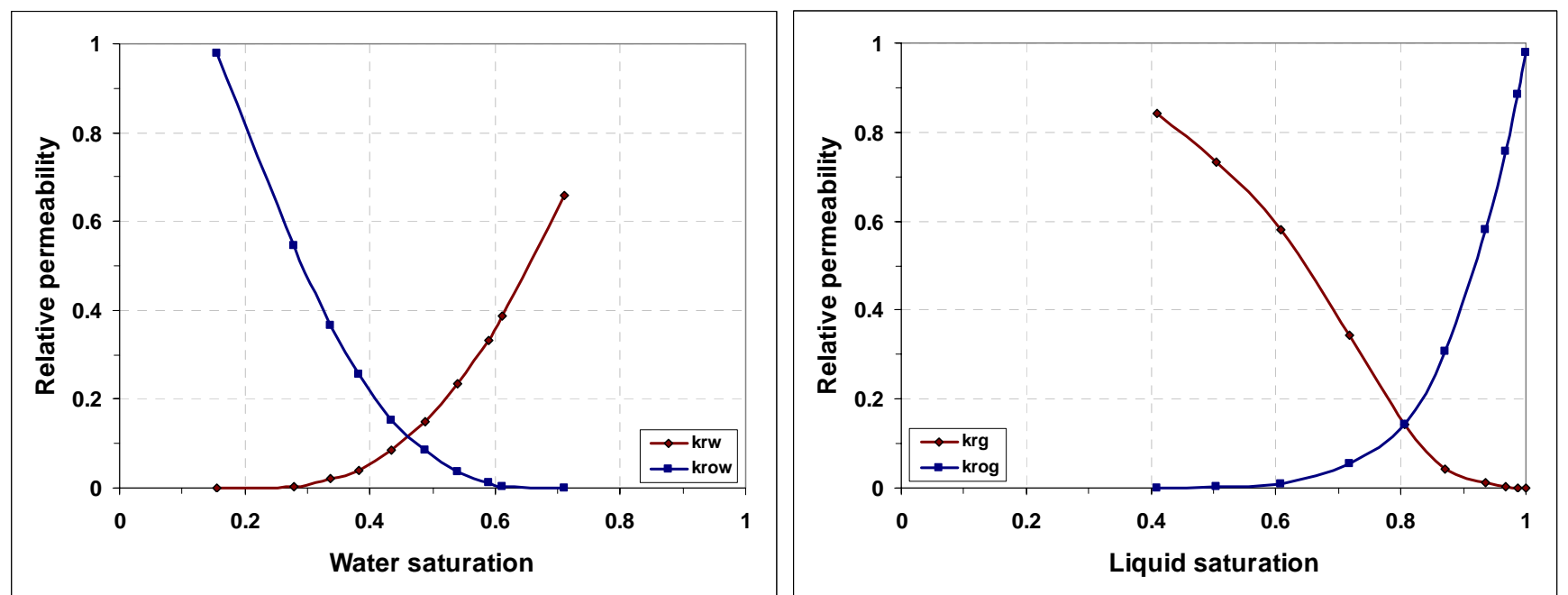

Fig. 4. Relative-permeability curves from the oil-wet Miocene Kareem Formation, UAE (Core Laboratories, 1982).
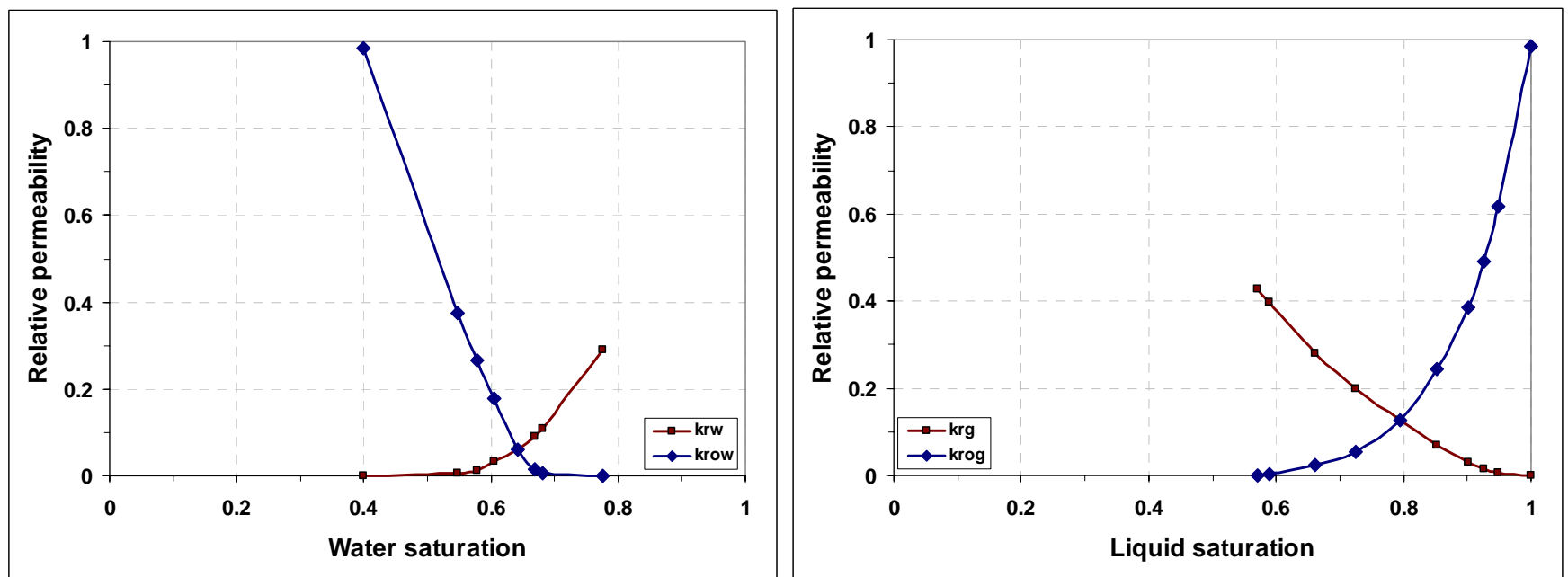

Fig. 5. Relative-permeability curves from the water-wet I-Sand Formation, Argentina (Core Laboratories, 1982). 


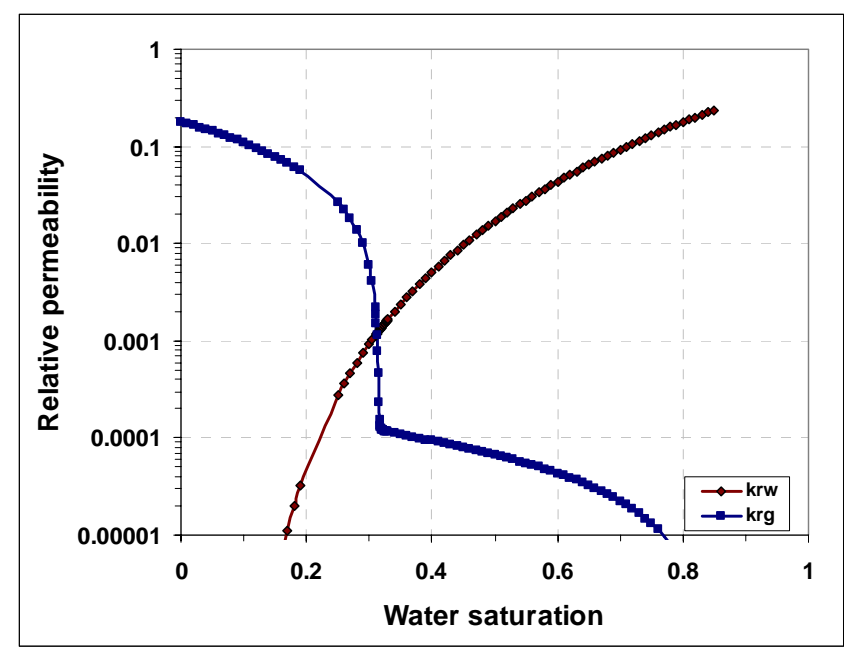

Fig. 6. Gas-water relative-permeability used in foam case, from Renkema and Rossen (2007). Effective gas relative permeability here accounts for all effects of foam on gas mobility. Foam greatly reduces gas mobility for water saturations above a threshold value, which here is $\mathbf{0 . 3 1 6}$. Note shift to log scale for relative permeabilities. An immobile oil saturation of 0.15 is assumed in this plot.

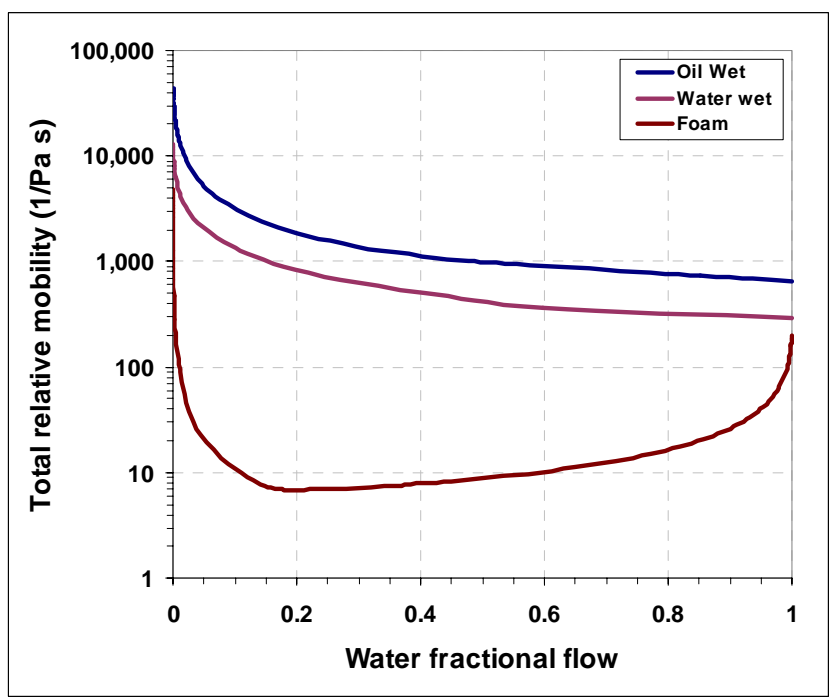

Fig. 7. Total relative mobility (Pa.s) ${ }^{-1}$ as a function of water fractional flow for each of the three sets of relative permeability curves in Figs. 4 to 6 . In both oil-wet and water-wet cases total relative mobility decreases monotonically with increasing water fraction. For foam, the lowest total relative mobility occurs at intermediate range of water fraction. The WAG simulations in this study correspond to an average injected water fraction of about $\mathbf{0 . 3 3}$.

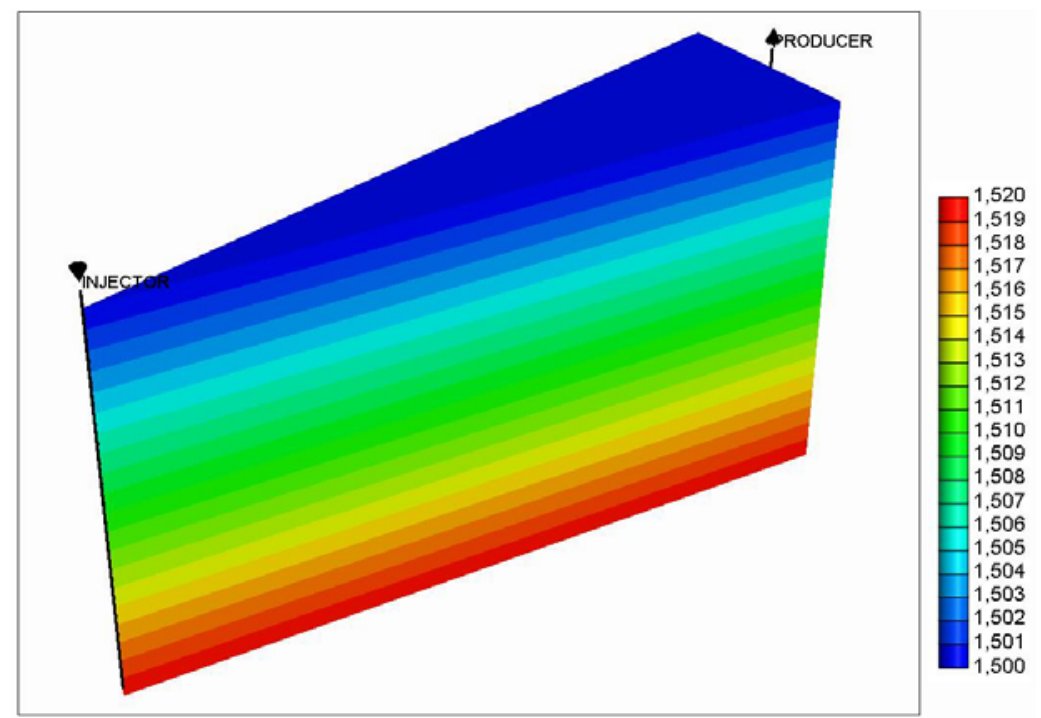

Fig. 8. 3D reservoir pay depth $(\mathrm{m})$ and well configuration for oil wet case. 


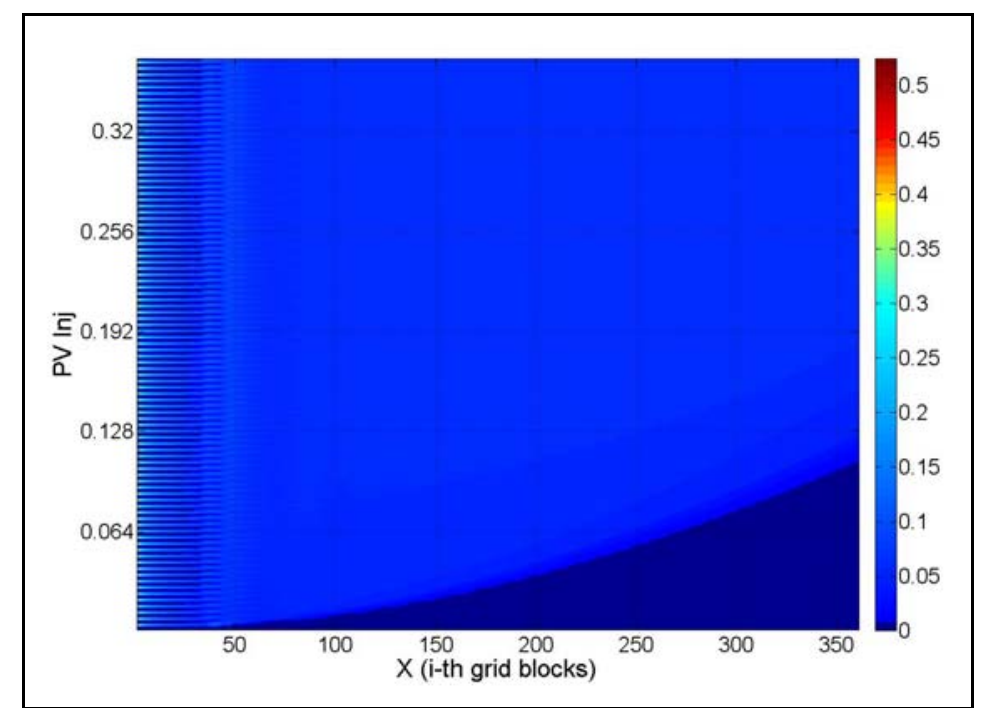

Fig. 9. Gas saturation as a function of position (grid block number) and time for the 1D case of oil-wet relative permeabilities. In this case a cycle of gas and water injection represents 0.004 PV.

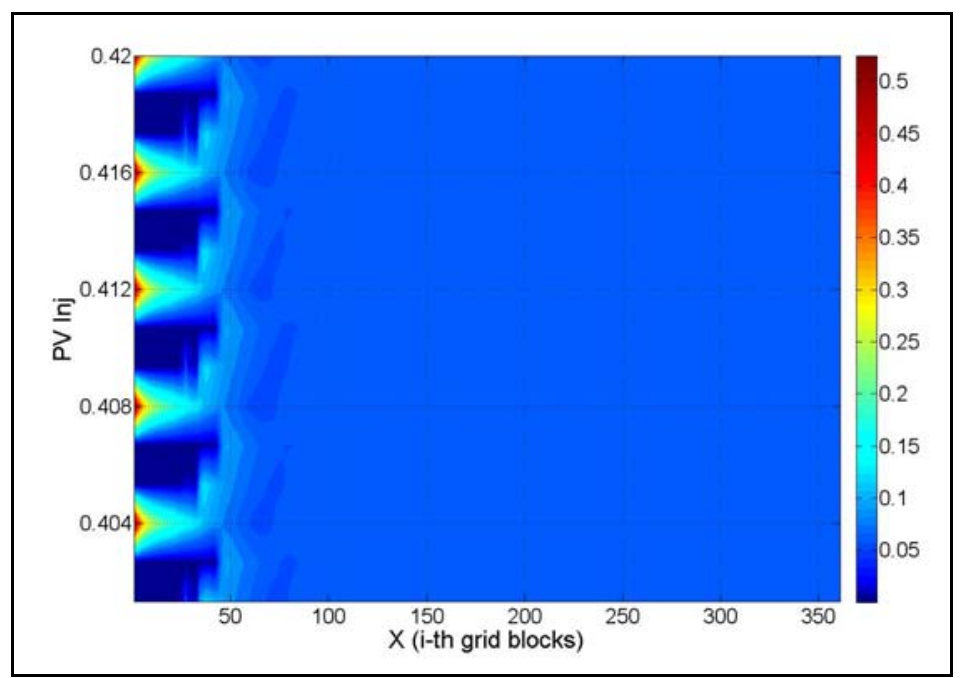

Fig. 10. Gas saturation as a function of location and time for 1D WAG injection, oil-wet case, after reaching steadily repeating behavior, for cycles of 0.004 PV. This figure represents Fig. 8 with an expanded time scale.

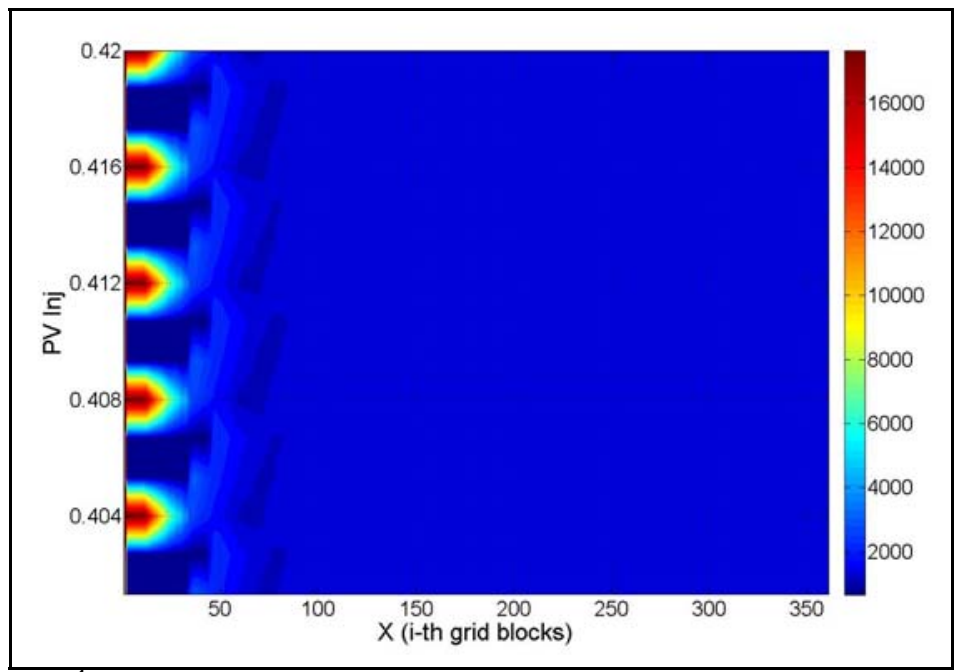

Fig. 11. Total relative mobility (Pa.s) ${ }^{-1}$ as a function of location and time for 1D WAG injection, oil-wet case, with cycles of 0.004 PV, at long times; cf. Fig. 9. 


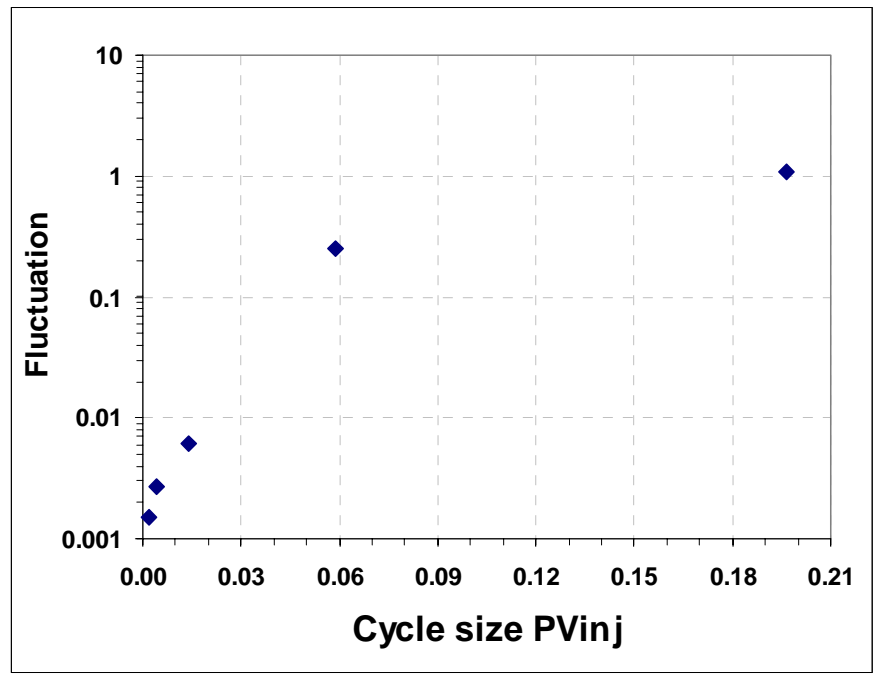

Fig. 12. Fluctuations in total relative mobility, which define as delta of maximum and minimum total relative mobility divided by the value of total relative mobility for co-injection, at the outer radius of the 1D reservoir as a function of cycle size for WAG processes in the oil-wet reservoir. Co-injection corresponds to zero fluctuation at vanishing slug size.

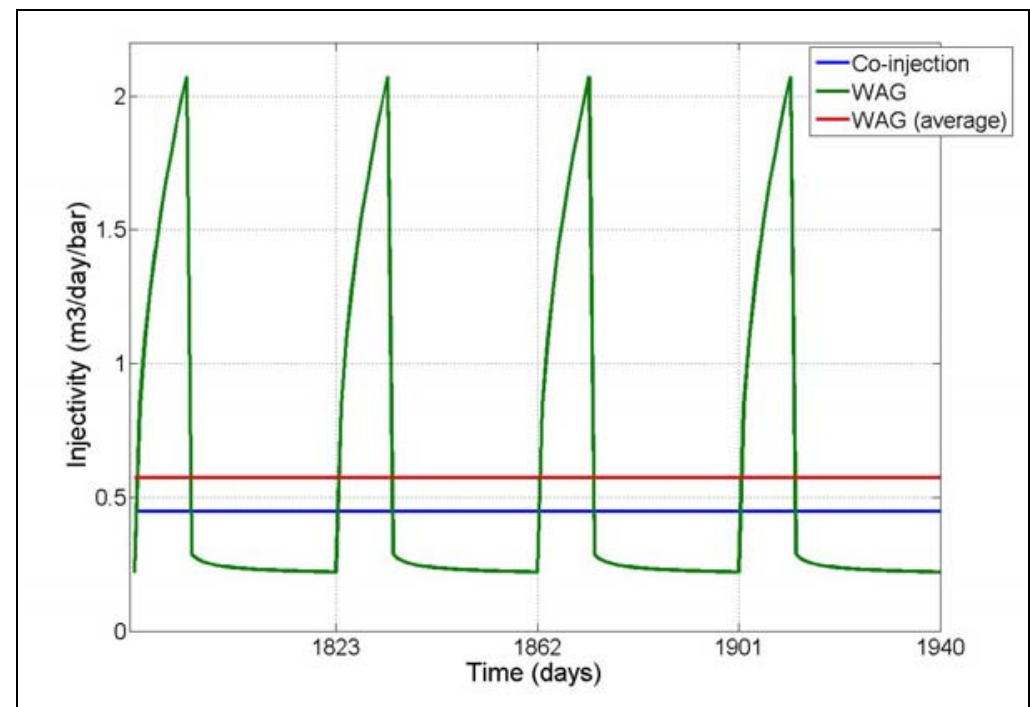

Fig. 13. Injectivity during cyclic WAG injection into the oil-wet reservoir, with 29 days water injection and 10 days gas injection (0.059 PVIcycle). The red line is average injectivity of the WAG process; the blue line, injectivity for co-injection. The WAG process shows an increase in average injectivity by a factor of 1.24 over co-injection.

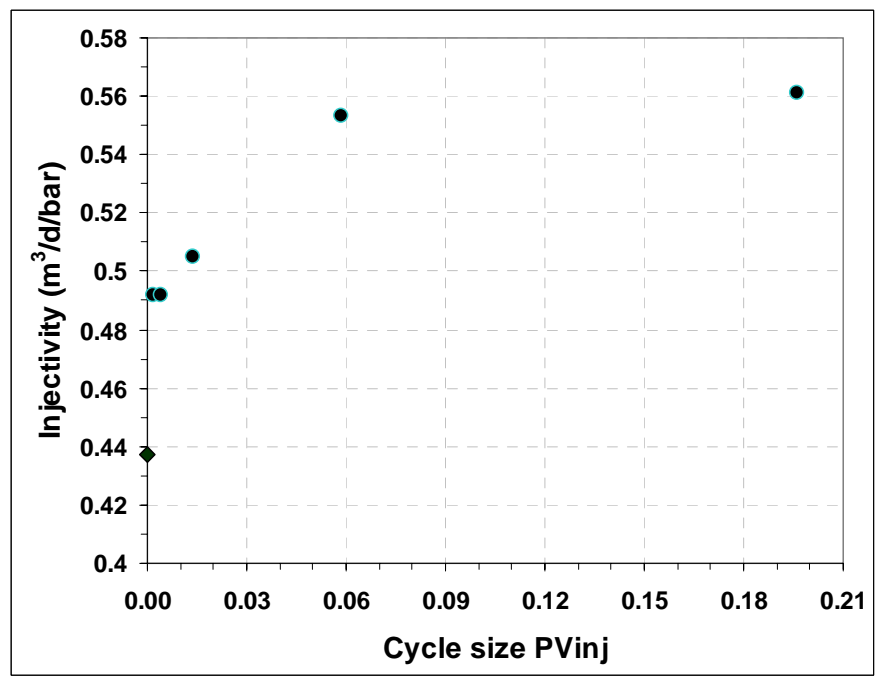

Fig. 14. Average injectivity in WAG injection as a function of cycle size in the oil-wet reservoir. 


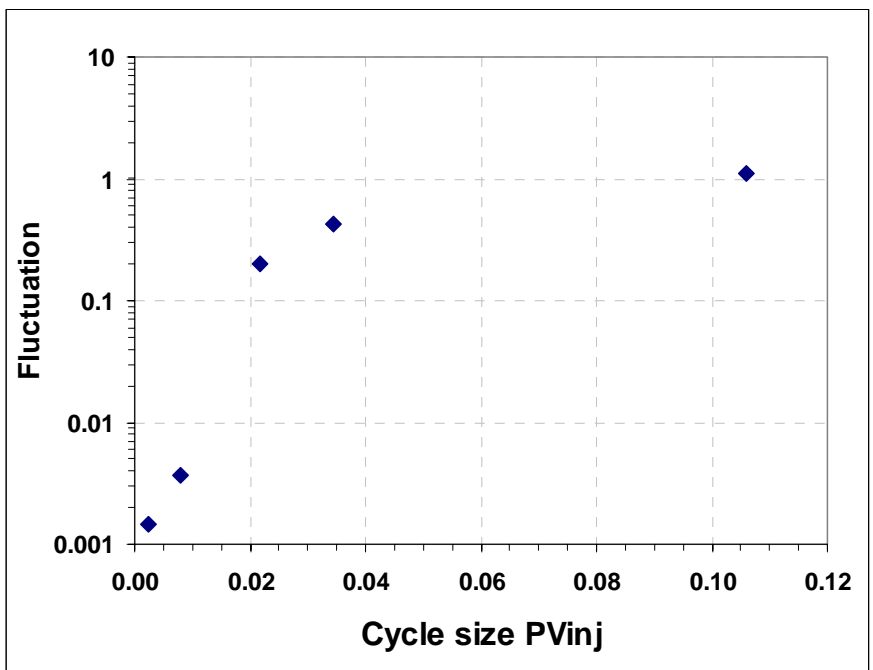

Fig. 15. Fluctuations in total relative mobility at the outer radius of the 1D reservoir, as a fraction of the value of total relative mobility for co-injection, as a function of cycle size for WAG processes in the water-wet reservoir. Co-injection corresponds to zero fluctuation at vanishing slug size.

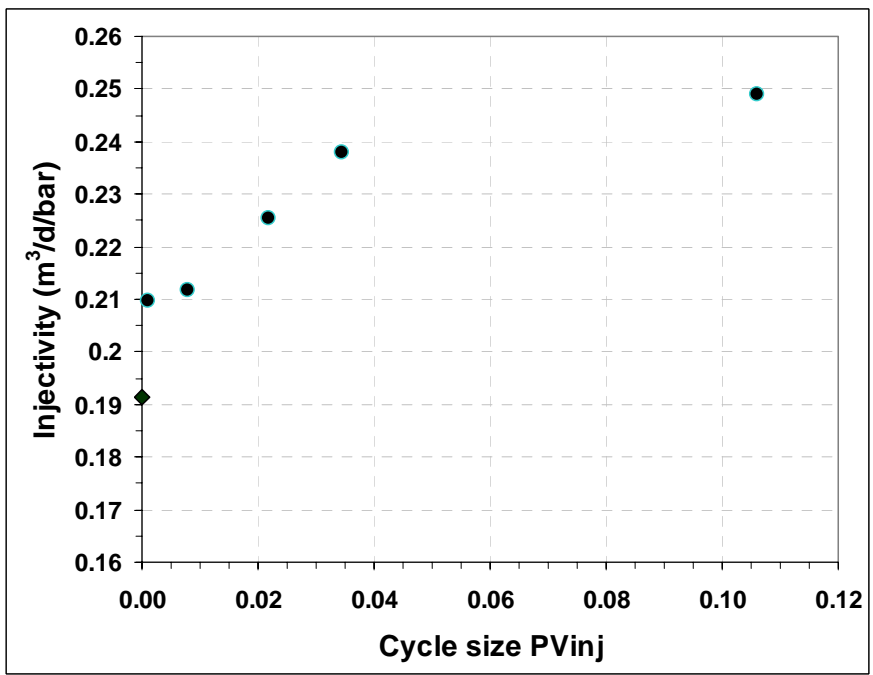

Fig. 16. Average injectivity in WAG injection as a function of cycle size in the water-wet reservoir.

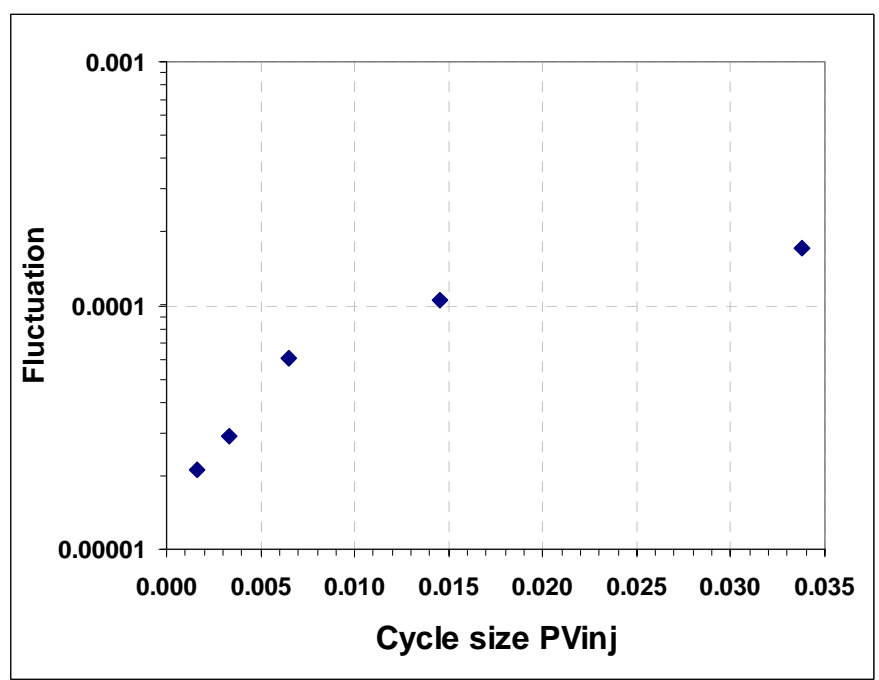

Fig. 17. Fluctuations in total relative mobility, at the outer radius of the 1D reservoir, as a fraction of the value of total relative mobility for co-injection, as a function of cycle size for SAG (foam) processes. Co-injection corresponds to zero fluctuation at vanishing slug size. 


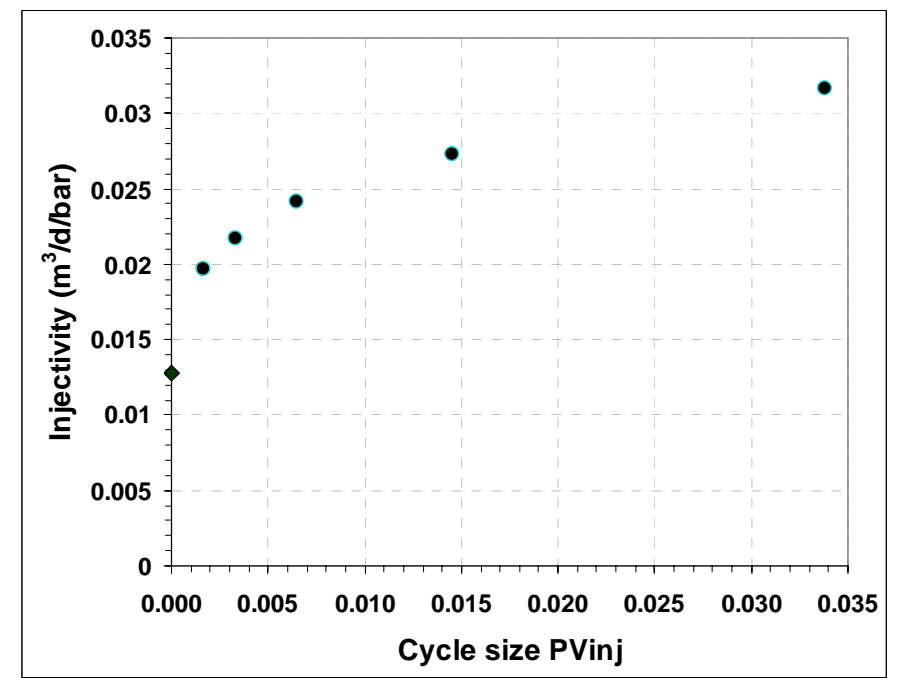

Fig. 18. Average injectivity in WAG injection as a function of SAG cycle size in the foam case.

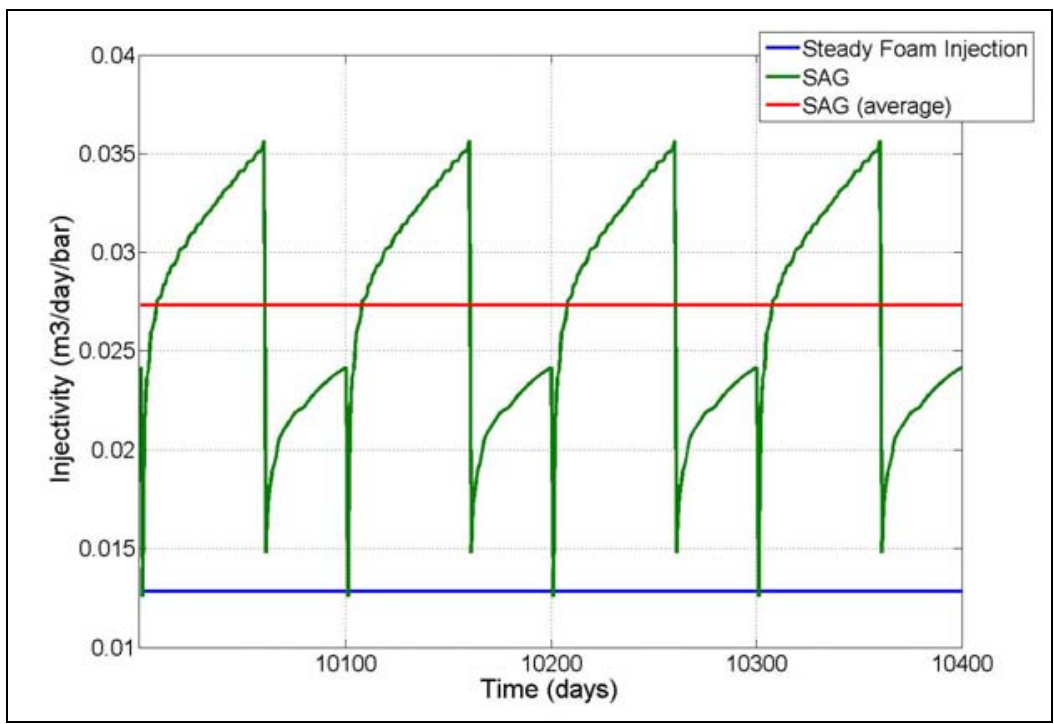

Fig. 19. Injectivity during SAG injection (40 days water injection and 60 days gas injection; total cycle size 0.015 PV) after steady, repeating pattern is attained, and steady injection of foam in 1D reservoir. The SAG process shows an increase in average injectivity by a factor of 2.13 over steady injection of foam.

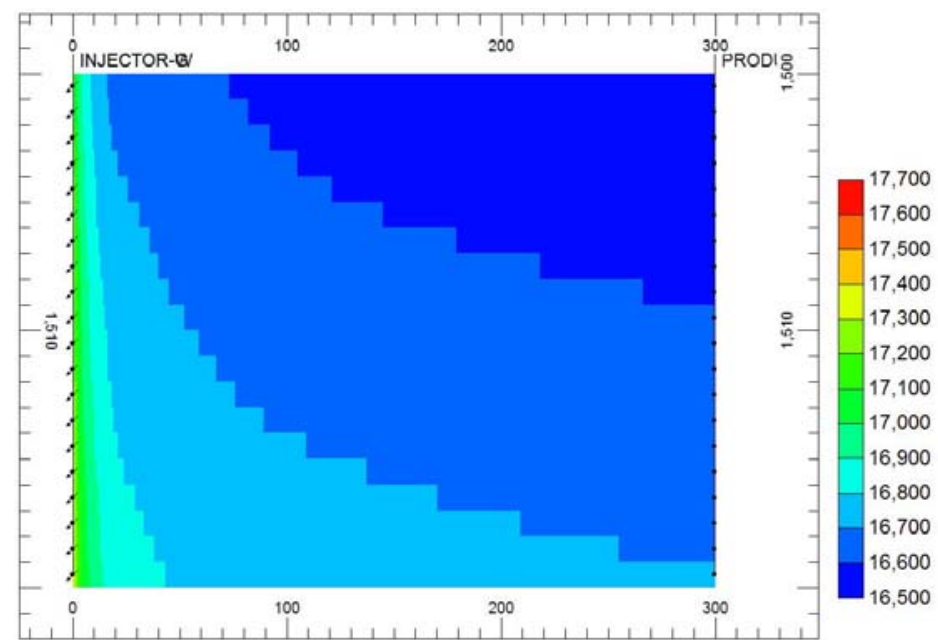

Fig. 20. Pressure distribution $(\mathrm{kPa})$ at the end of water injection, cycle size $0.106 \mathrm{PV}$, water-wet reservoir. Pressure is largely dissipated near the wellbore. 


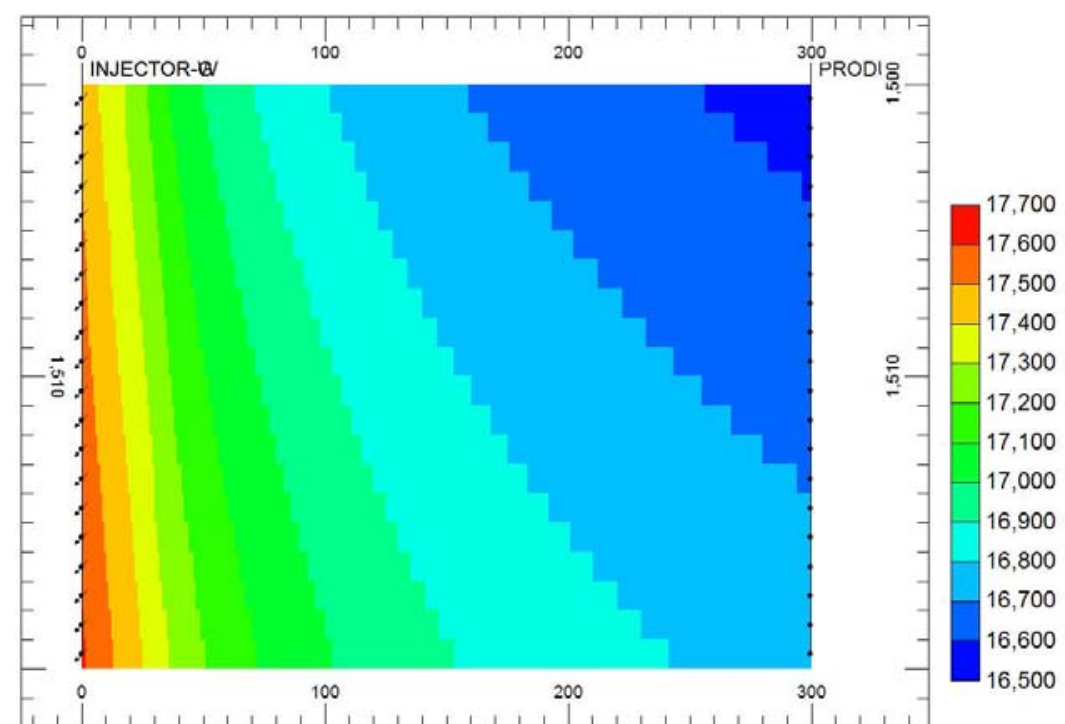

Fig. 21. Pressure distribution (kPa) at the end of gas injection, cycle size $0.106 \mathrm{PV}$, water-wet reservoir. Injection pressure is dissipated over wider portion of the reservoir than in co-injection.

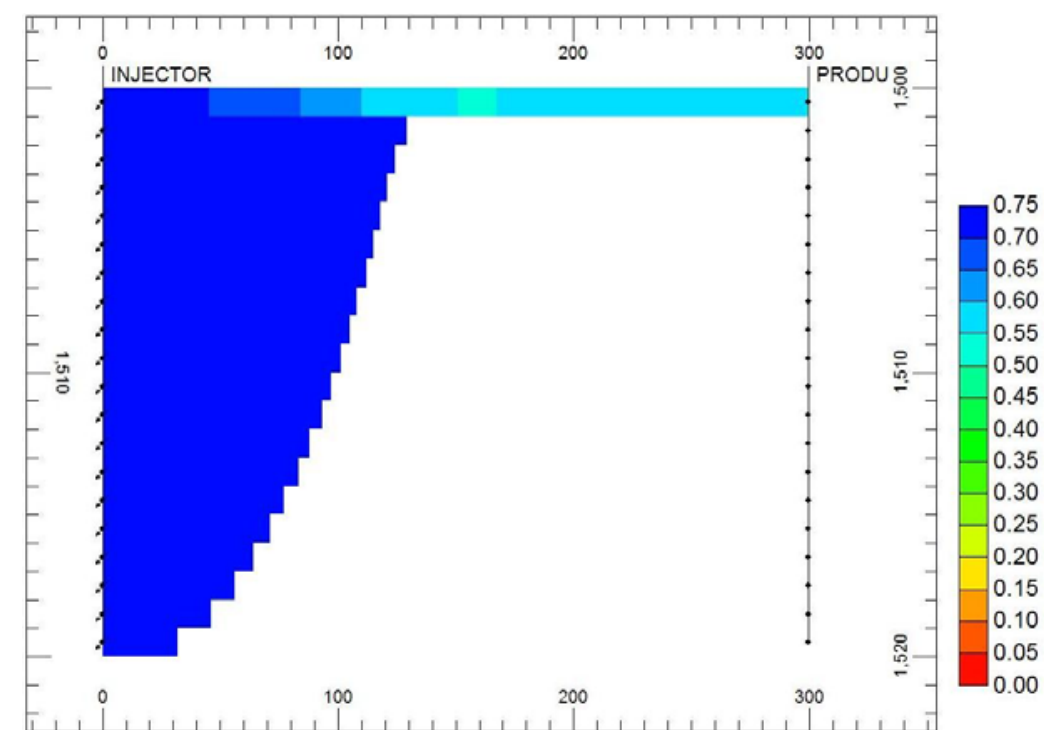

Fig. 22. Water-saturation profile for co-injection in water-wet reservoir; $R_{g}=125 \mathrm{~m}$.

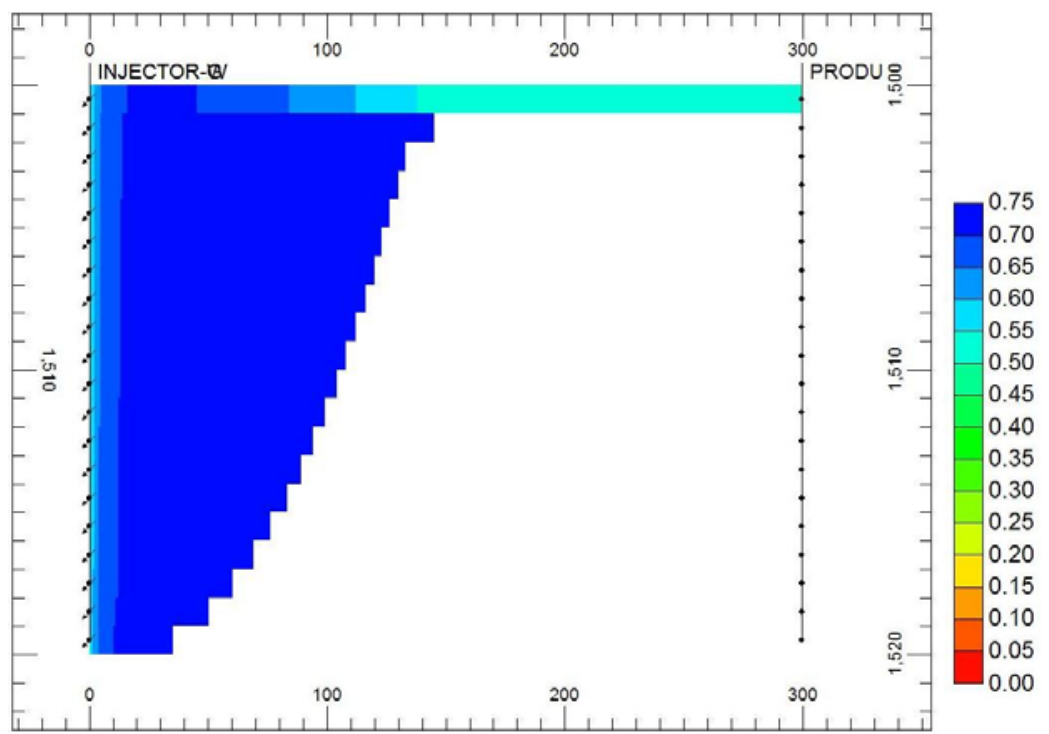

Fig. 23. Water saturation at the end of gas injection in WAG in water-wet reservoir with cycle size of $0.001 \mathrm{PV} ; \boldsymbol{R}_{g}=136 \mathrm{~m}$. 


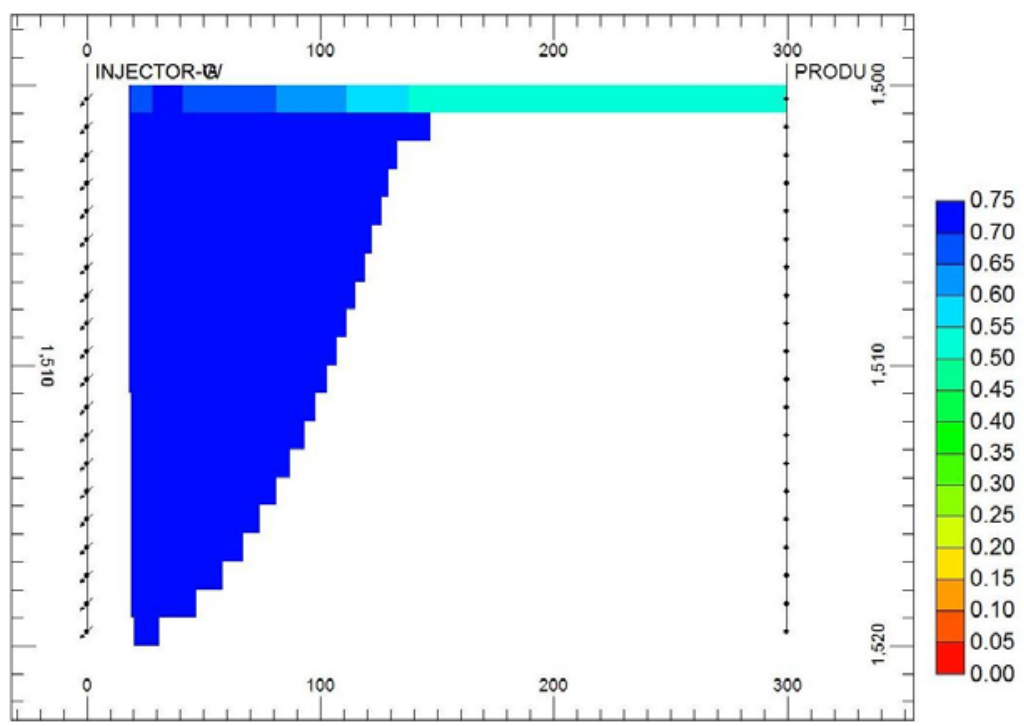

Fig. 24. Water saturation at the end of water injection in WAG in water-wet reservoir with cycle size of $0.001 \mathrm{PV} ; \boldsymbol{R}_{g}=138 \mathrm{~m}$.

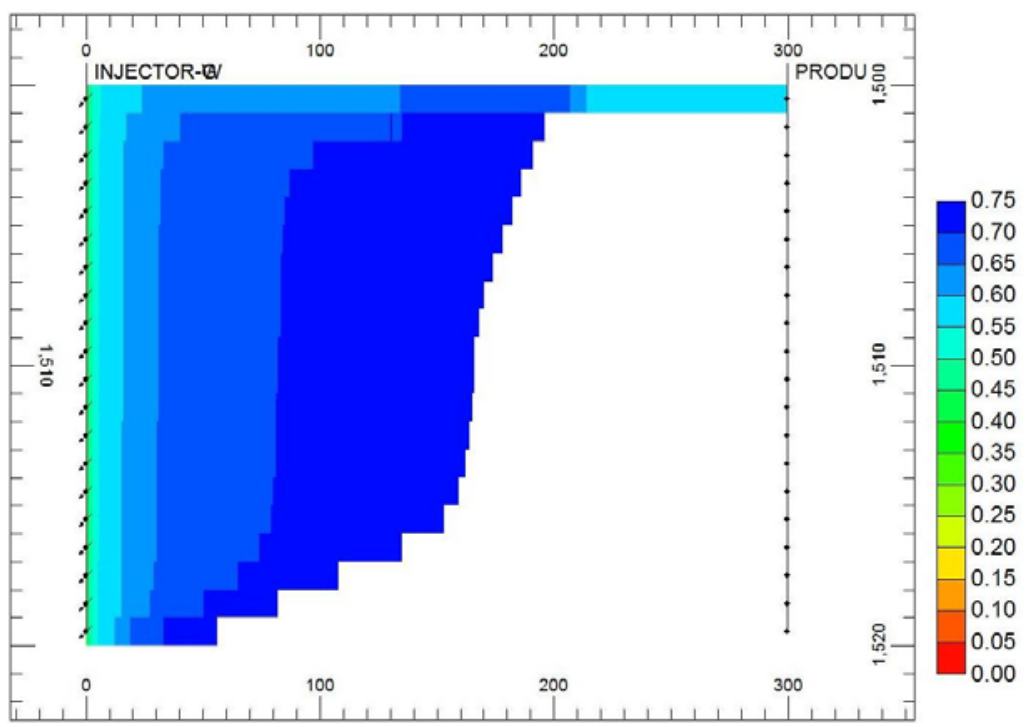

Fig. 25. Water saturation at the end of gas injection in WAG in water-wet reservoir with cycle size of $0.034 \mathrm{PV} ; \boldsymbol{R}_{g}=200 \mathrm{~m}$.

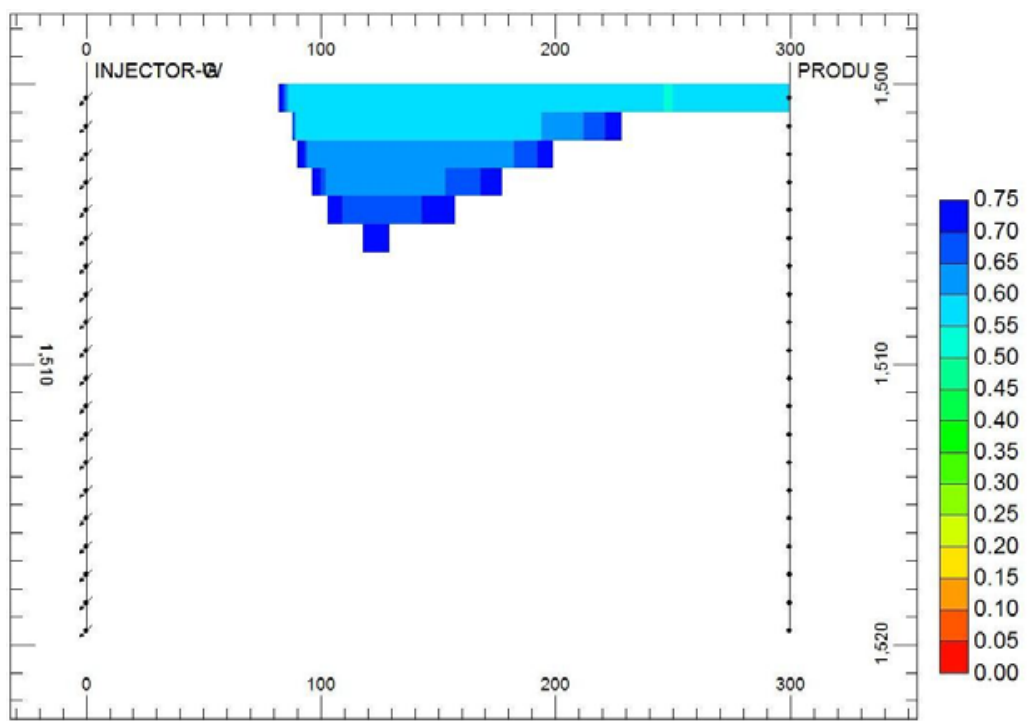

Fig. 26. Water saturation at the end of water injection in WAG in water-wet reservoir with cycle size of $0.034 \mathrm{PV} ; \boldsymbol{R}_{g}=225 \mathrm{~m}$. 


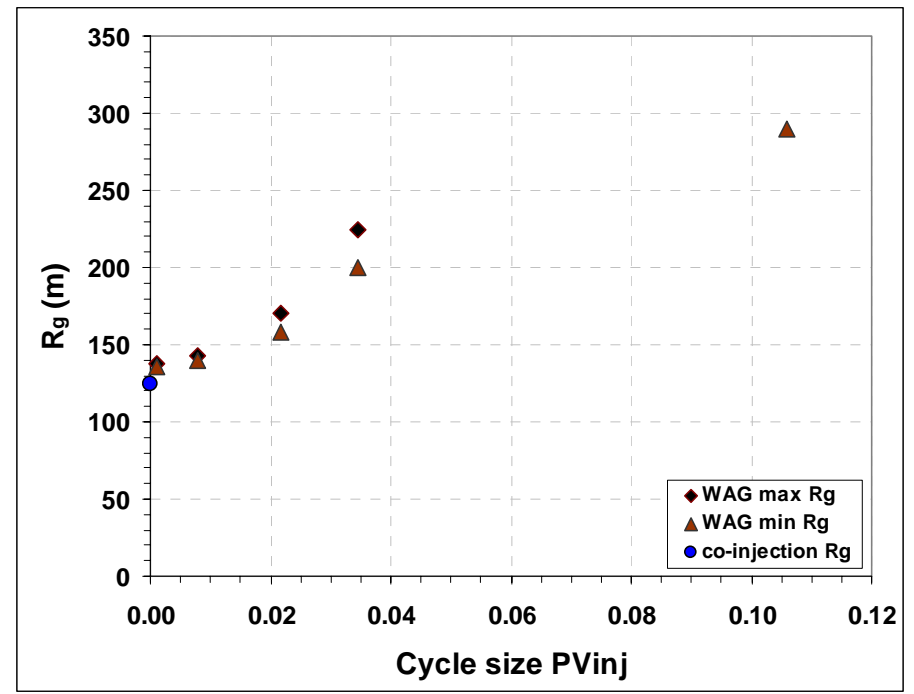

Fig. 27. Maximum and minimum values of $R_{g}$ as a function of WAG cycle size in water-wet reservoir. No maximum $\boldsymbol{R}_{g}$ is shown because segregation happens beyond the production well at $300 \mathrm{~m}$.

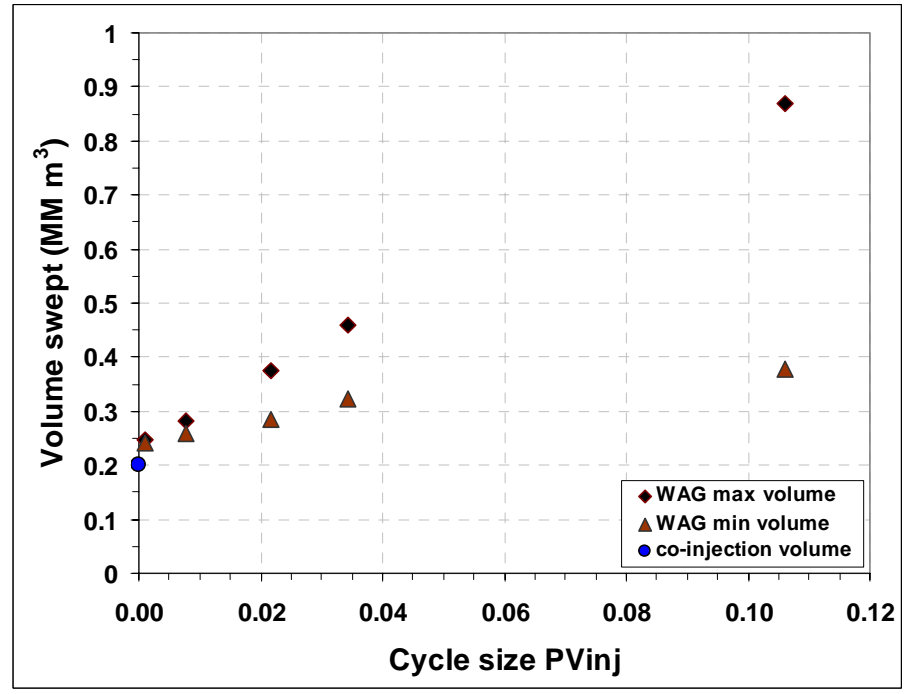

Fig. 28. Maximum and minimum volume of gas in reservoir as function of WAG cycle size in 2D water-wet case.

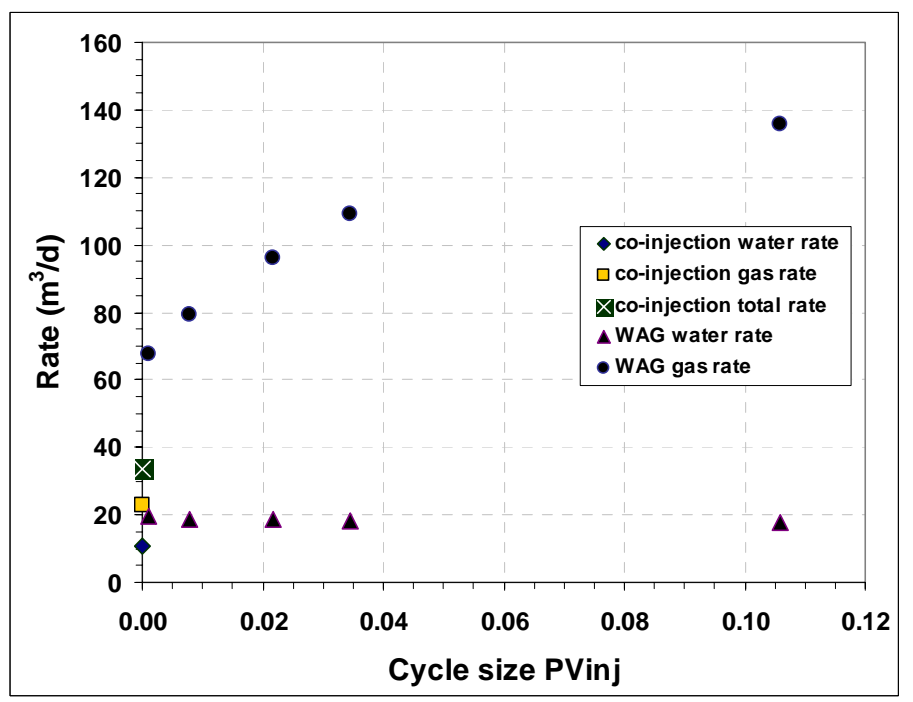

Fig. 29. Maximum and minimum injection rate as a function of WAG cycle size in 2D water-wet case. 


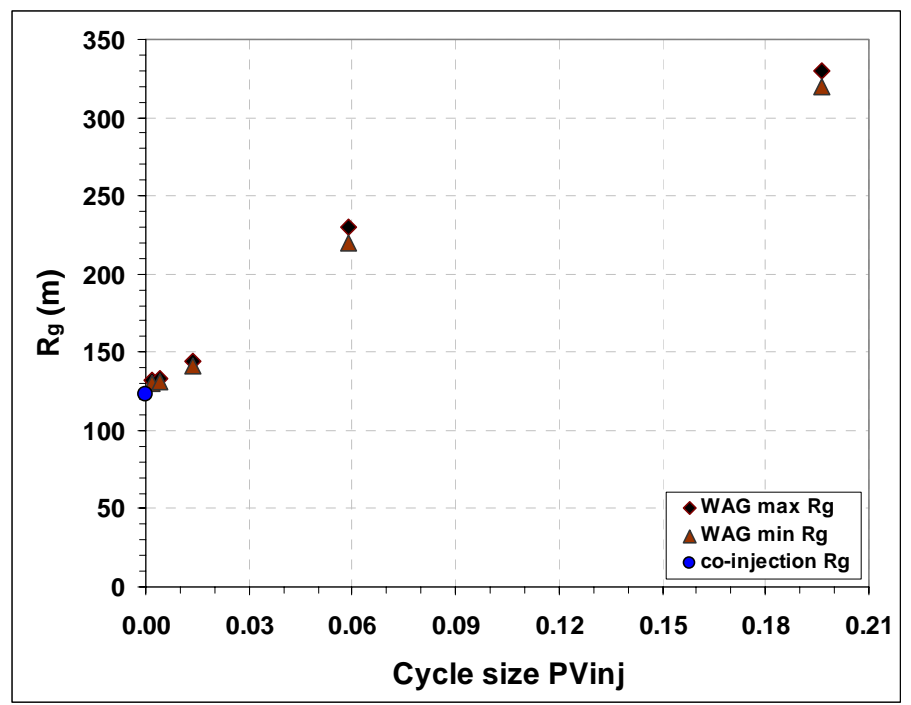

Fig. 30. Maximum and minimum values of $R_{g}$ as a function of WAG cycle size in oil-wet case.

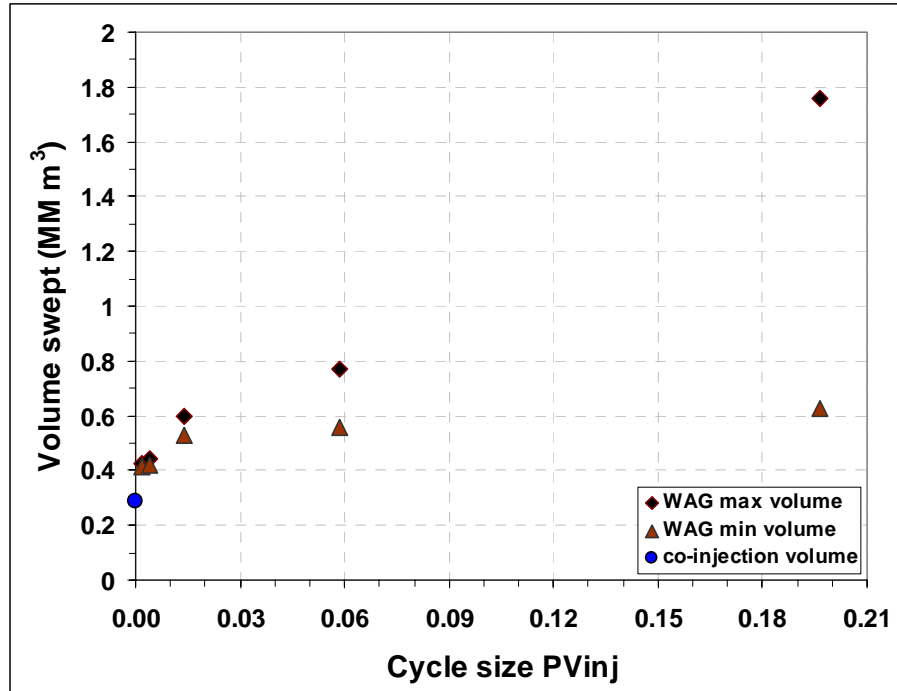

Fig. 31. Maximum and minimum volume of gas in reservoir as function of WAG cycle size in 2D oil-wet case.

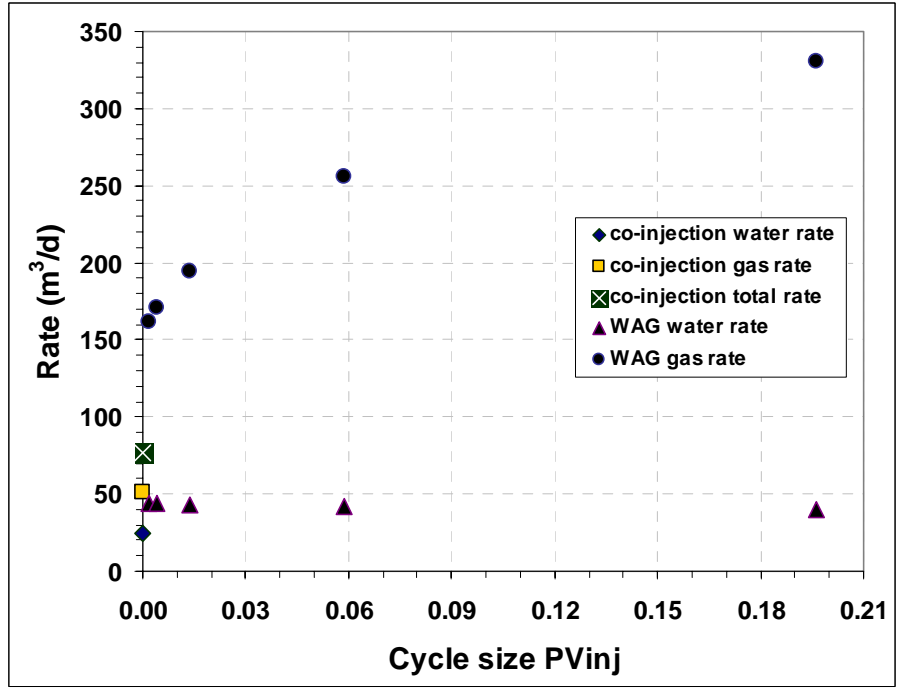

Fig. 32. Maximum and minimum injection rate as a function of WAG cycle size in 2D oil-wet case. 


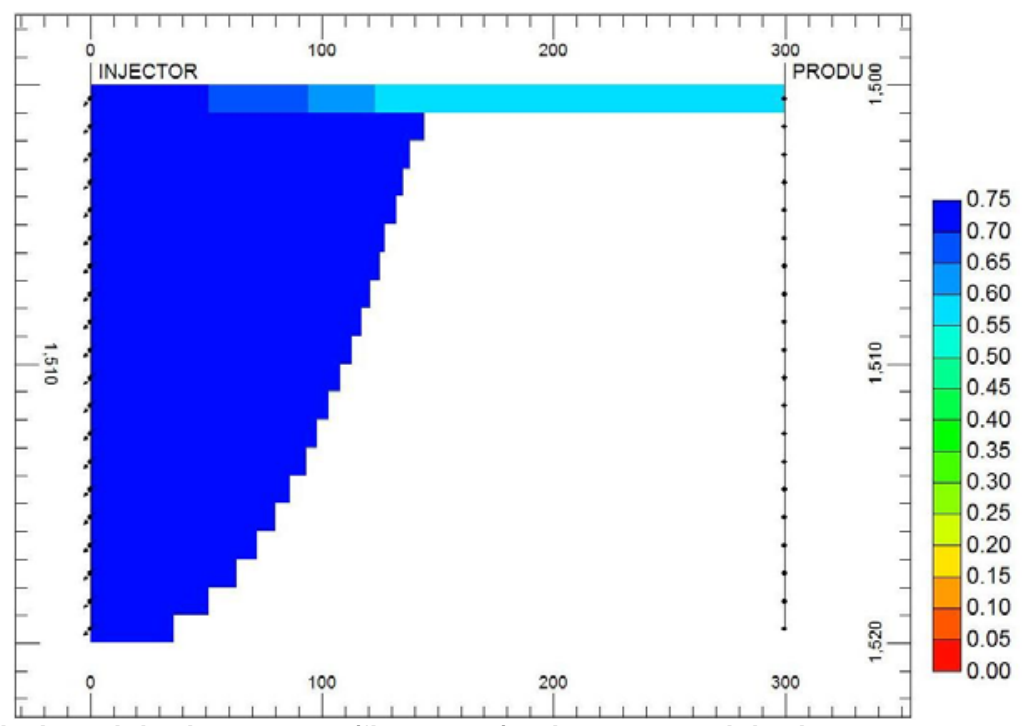

Fig. 33. Water-saturation profile in co-injection process (fixed WGR) using constant injection rate set to the average injection rate of WAG with 0.008 PV cycle: $R_{g}=140 \mathrm{~m}$.

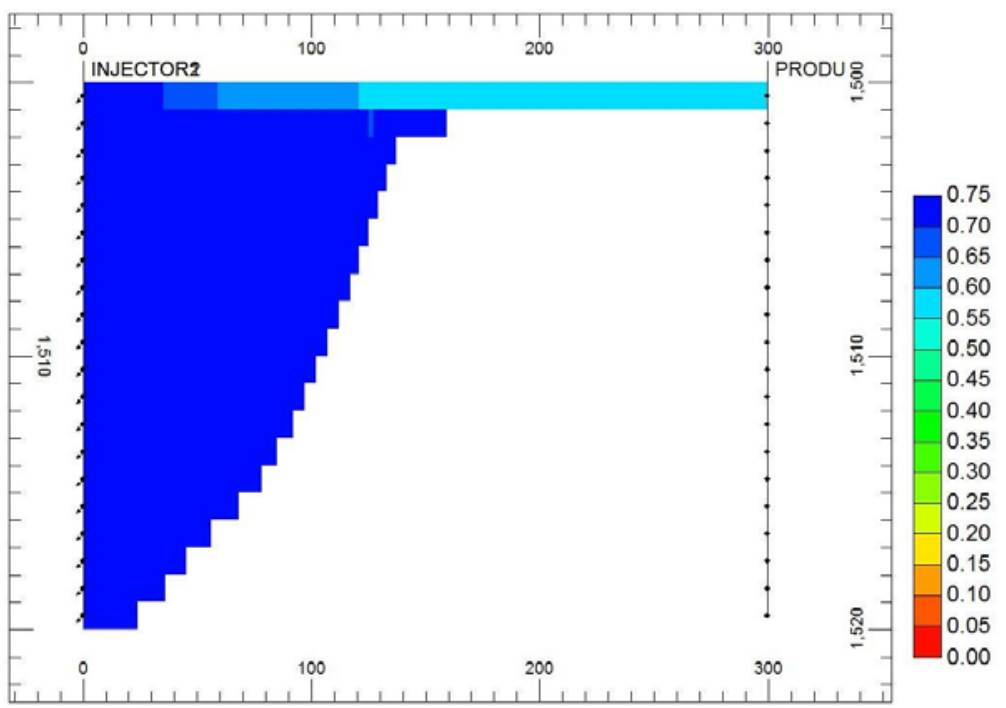

Fig. 34. Water-saturation profile showing minimum $R_{g}=141 \mathrm{~m}$ in co-injection process using injection-rate history of WAG with 0.008 PV cycle and fixed water fractional flow.

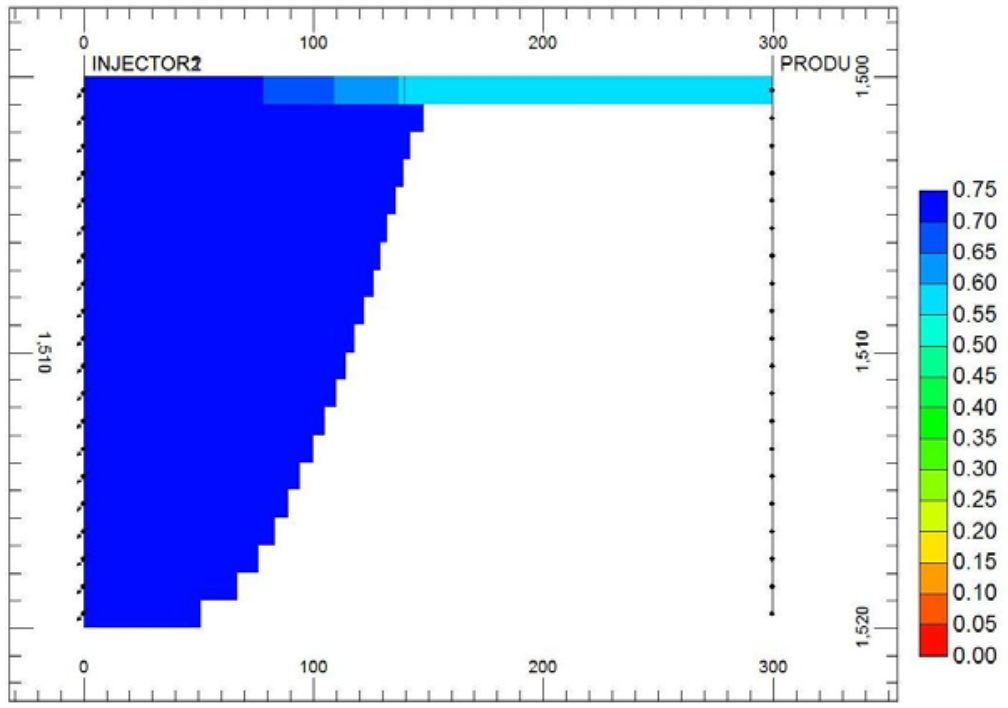

Fig. 35. Water-saturation profile showing maximum $R_{g}=148 \mathrm{~m}$ in co-injection process using injection-rate history of WAG with 0.008 PV cycle and fixed water fractional flow. 


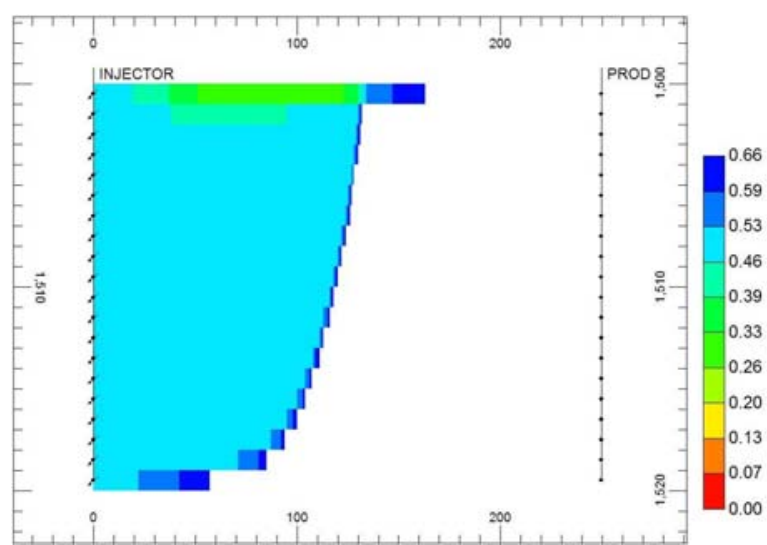

Fig. 36. Water-saturation profile for co-injection of foam; $R_{g}=132 \mathrm{~m}$.

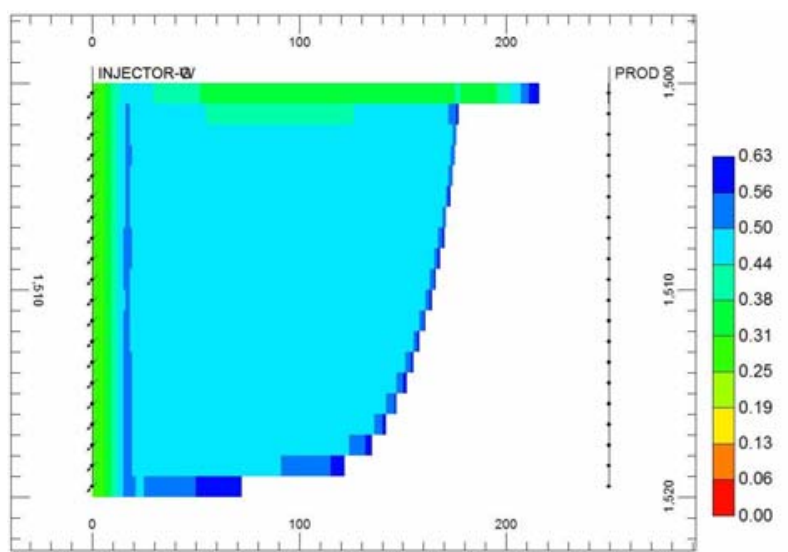

Fig. 37. Water saturation at the end of gas injection in SAG with cycle size of $0.002 \mathrm{PV} ; R_{g}=178 \mathrm{~m}$. This radius of segregation is indistinguishable from that at the end of water injection.

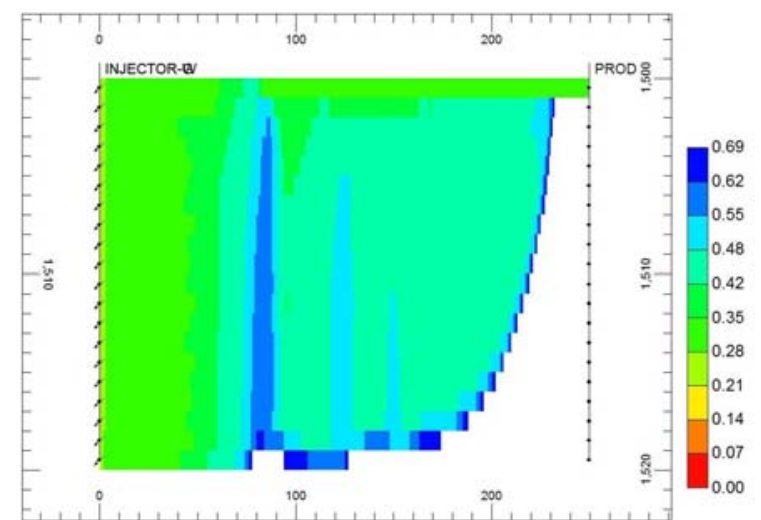

Fig. 38. Water saturation at the end of gas injection in SAG in water-wet reservoir with cycle size of $0.034 \mathrm{PV} ; \boldsymbol{R}_{g}=233 \mathrm{~m}$.

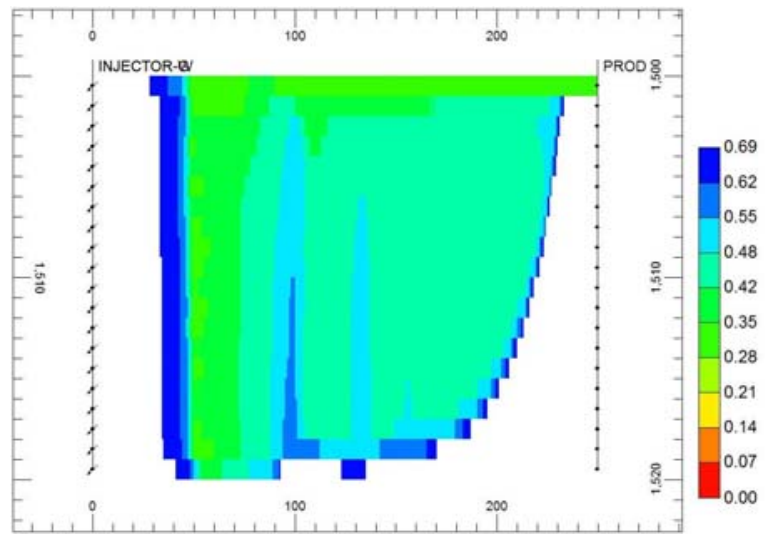

Fig. 39. Water saturation at the end of water injection in SAG in water-wet reservoir with cycle size of $0.034 \mathrm{PV} ; \boldsymbol{R}_{g}=233 \mathrm{~m}$. 


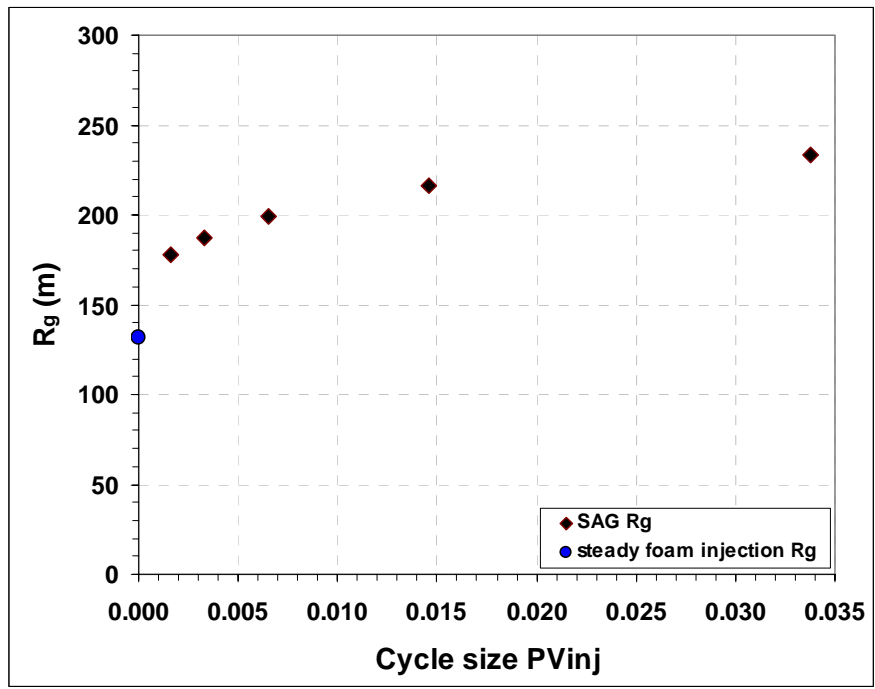

Fig. 40. $R_{g}$ as a function of SAG cycle size in foam case. We find virtually no variation in $R_{g}$ values with time for these cycle sizes.

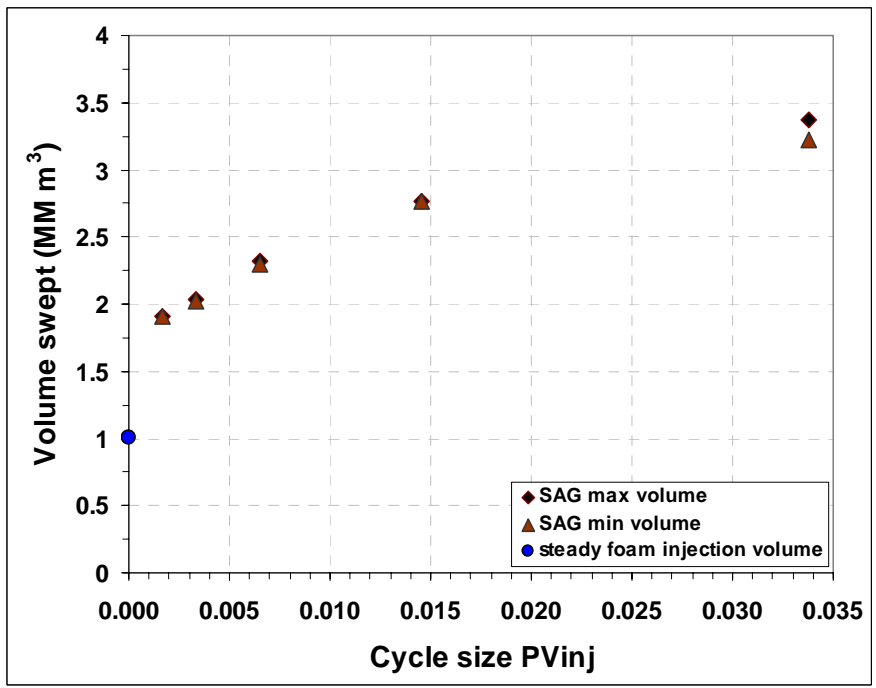

Fig. 41. Maximum and minimum volume of gas in reservoir as function of slug size in 2D foam case.

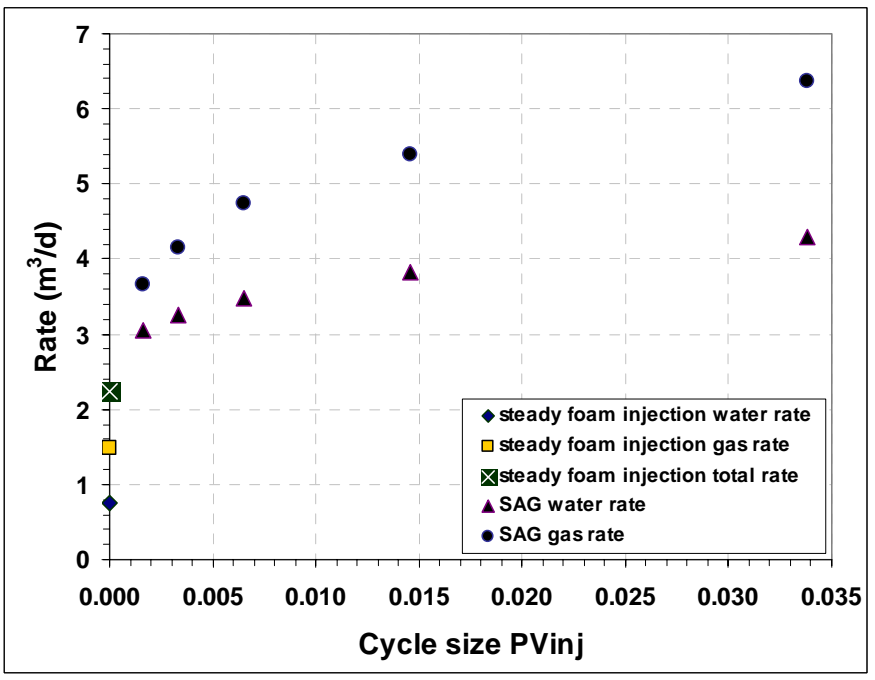

Fig. 42. Maximum and minimum injection rate as a function of SAG cycle size in 2D foam case. 